\title{
New porous medium Poisson-Nernst-Planck equations for strongly oscillating electric potentials
}

\author{
M. Schmuck ${ }^{1}$ \\ Departments of Chemical Engineering and Mathematics, Imperial College, \\ London $S W^{\text {Y }}$ 2AZ, UK, a)
}

(Dated: 2 November 2018)

We consider the Poisson-Nernst-Planck system which is well-accepted for describing dilute electrolytes as well as transport of charged species in homogeneous environments. Here, we study these equations in porous media whose electric permittivities show a contrast compared to the electric permittivity of the electrolyte phase. Our main result is the derivation of convenient low-dimensional equations, that is, of effective macroscopic porous media Poisson-Nernst-Planck equations, which reliably describe ionic transport. The contrast in the electric permittivities between liquid and solid phase and the heterogeneity of the porous medium induce strongly oscillating electric potentials (fields). In order to account for this special physical scenario, we introduce a modified asymptotic multiple-scale expansion which takes advantage of the nonlinearly coupled structure of the ionic transport equations. This allows for a systematic upscaling resulting in a new effective porous medium formulation which shows a new transport term on the macroscale. Solvability of all arising equations is rigorously verified. This emergence of a new transport term indicates promising physical insights into the influence of the microscale material properties on the macroscale. Hence, systematic upscaling strategies provide a source and a prospective tool to capitalize intrinsic scale effects for scientific, engineering, and industrial applications.

\footnotetext{
a) Electronic mail: m.schmuck@imperial.ac.uk.
} 
New porous medium Poisson-Nernst-Planck equations

\section{INTRODUCTION}

The Poisson-Nernst-Planck (PNP) equations can be applied in many different physical contexts such as modeling of ionic transport, e.g. batteries, supercapacitors, fuel cells, and capacitive desalination devices. Especially the fields of electrokinetics and electrohydrodynamics gained an increasing interest in recent years. Actual research aims to take advantage of scale effects in micro- and nano-fluidic devices for industrial applications and for the creation of chip-like devices ("lab on a chip"). Such devices can perform separation, mixing, and chemical analysis tasks. It is also possible to design electrokinetic pumps..$^{\underline{1}}$

The study of geometric effects on the scale of cell membranes, muscles, and neurons by means of PNP equations finds currently a lot of attention in biology and medcine..$^{2} \cdot \underline{3}$ The essential goal is to better understand how calcium ions, i.e. $\mathrm{Ca}^{2+}$-ions, move in voltagedependent calcium channels for example. These channels are a group of voltage-gated ion channels which can be found in muscles, glial cells, and neurons. Recent research attempts to mimic such biological ion channels with synthetically built channels. $\stackrel{4}{=}$ For example by modifying channel geometry and surface charge one tries to better understand the effect of rectification. Rectificiation can be descriptively explained by the comparison of ionic flux with an electric current through a pn-diode. One usually studies rectfication factors (ratio of forward current to reverse current) in this context, see 5 for example.

This broad range of applications in heterogeneous environments strongly rely on models which reliably and systematically account for effects of the microscale on the macroscale. A very common approach for deriving effective macroscopic equations is volume averaging. $\underline{\underline{6}} \underline{\underline{8}}$ Unfortunately, it is still unclear how to systematically treat nonlinear terms by this intuitive method. A technically slightly more involved approach is the homogenization $\operatorname{method}^{-} \underline{-11}$ which provides a reliable and systematic alternative under the assumption of a periodic pore distribution.

The general importance and the strong demand of properly upscaled equations in engineering, and design as well as optimization of scientific and industrial devices call for mathematical tools that rely on well-established principles for multiscale problems. Here, we want to systematically extend the widely accepted PNP system from the free space case towards solid-electrolyte composites showing a high contrast between its electric permittivities. For this purpose, we consider the full PNP equations with the help of a modified 
asymptotic two-scale expansion. This new approach accounts for the nonlinear and coupled structure of the system, see Theorem III.2. As our main result, we derive the following new effective macroscopic porous medium PNP equations, that is,

$$
\begin{cases}p \partial_{t} \mathrm{u}_{0}^{r}-p \Delta \mathrm{u}_{0}^{r}+\operatorname{div}\left(\mathbb{D}^{r}(t, x) \nabla \mathrm{u}_{0}^{3}\right)-\operatorname{div}\left(z_{r} \mathrm{u}_{0}^{r} \mathbb{M} \nabla \mathrm{u}_{0}^{3}\right)=0 & \text { in } \Omega_{T} \\ -\operatorname{div}\left(\boldsymbol{\epsilon}^{0} \nabla \mathrm{u}_{0}^{3}\right)=p\left(\mathrm{u}_{0}^{1}-\mathrm{u}_{0}^{2}\right) & \text { in } \Omega_{T}\end{cases}
$$

which is valid under local (pore level) thermodynamic equilibrium and for arbitrary Debye lengths $\lambda_{D}>0$. The parameter $p$ denotes the porosity and $\mathbb{D}^{r}, \mathbb{M}$, and $\boldsymbol{\epsilon}^{0}$ are effective transport tensors defined by the upscaling subsequently performed. The variable $\mathrm{u}_{0}^{r}$ represents effective macroscopic quantities such as the concentration of positively charged ions $n^{+}$for $r=1$, the density of negatively charged ions $n^{-}$for $r=2$, and the electrostatic potential $\Phi$ for $r=3$.

All equations appearing during the upscaling are rigorously justified by well-posedness criteria. In particular, Lemma II.4 (Section 【II) guarantees the solvability of the new system (1) which shows a new term $\mathbb{D}^{r}$ that accounts for a dominant influence of the oscillating electric potential on the concentrations. We emphasize that this new term emerges as a result of an adapted asymptotic multi-scale expansion introduced in order to account for the heterogeneity induced by the porous medium and by the electric permittivities. That means, in the classical asymptotic expansion

$$
u_{s}(x)=u_{0}(x, x / s)+s u_{1}(x, x / s)+s^{2} u_{2}(x, x / s)+\ldots,
$$

we assume a special separation between the micro- and macro-scale in the terms $u_{1}$ and $u_{2}$ in difference to related literature ${ }^{12}-\underline{15}$ and classical homogenization theory, $\stackrel{10,16}{\underline{16}}$ see (24) in Theorem $\amalg I .2$ below. We point out that the nonlinear character of the Nernst-Planck equations leads with (2) to ill-posed reference cell problems without additional assumptions. Hence, we suppose that the reference cells, which define the micro-geometry of the porous medium, are in thermodynamic equilibrium. This guarantees then well-posedness. This solvability issue might also be the reason why the upscaling of the PNP equations was mainly restricted to thin double layer type approximations $\frac{17,18}{18}$ or linearized formulations in the context of Onsager reciprocal relations ${ }^{27}, 28$ so far. $\stackrel{12,13}{\underline{2}}$ Physically, such situations occur when the electric permittivities between the electrolyte and the solid material are far apart, see Section $1 \mathrm{~A}$. 
New porous medium Poisson-Nernst-Planck equations

The article is organized in the following way: The dominant oscillating behavior of the electric potential is motivated by the contrast in the electric permittivities between the solid and the liquid phase in Section $[\mathrm{I}$ where also a related effective media theory is discussed. A historical overview of closely related upscaling results is given in Section IB. In Section II, we state elementary results and introduce necessary notation. The main results follow in Section III. Finally, we prove all results in Section IV.

\section{A. Physical motivation: Dielectric permittivities of solids and liquids}

In this section, we state the physical setting which leads to strongly oscillating electric potentials in composites such as a porous medium permeated by a dilute electrolyte. A related example where such oscillations are well-known is the electric field over a material with strongly heterogeneous conductivities. This is also one of the classical fields of effective media theory $\underline{\underline{19}}$ and homogenization theory ${ }^{\underline{9}} \underline{\underline{10}}$ where one often assumes a periodic representation of the heterogeneities for simplicity. In fact, the high-frequency electric permittivity and the low-frequency electric conductivity are formally equivalent because of the equivalence in the governing equations. However, the situation for the PNP system here is slightly different since we have to deal with a non-linearly coupled system of equations. As explained previously, we account for this difference by a non-standard asymptotic expansion that factors the strong influence of the electric potential in. Moreover, the equivalence between permittivity and conductivity implies that their mathmatical computation is equivalent.

As in the case of conductivity, the strength of the oscillations can be controlled by the distance between the different electric permittivities for our problem here. Since we study a dilute electrolyte, we can expect an electric permittivity of the liquid phase to be around 80 at room temperature and a frequency under $1 k H z$ (of course, this also depends on the electrolyte employed). For the solid phase, we can expect an electric permittivity between 2 and 5, i.e., paper 3, alumina 4.5, teflon 2.1, porcelain 5.1, and plexiglas 2.8. But in many fields, a systematic derivation of effective media quantities such as the electric permittivity is still lacking. ${ }^{20}$ Our subsequently derived equations reliably define such an effective electric permittivity for periodic porous media. We emphasize the importance of characterizing

porous materials with respect to dielectric properties in microelectronics. 21 Moreover, we believe that a systematic and reliable upscaling of such complex composites using geometric 
New porous medium Poisson-Nernst-Planck equations

and material properties together with experimental validation gives promising perspectives for new scientific, technological, and industrial applications.

\section{B. Review of related upscaling results of the PNP equations}

We briefly give a shortened historical overview, see Table I, and point out differences of contributions mainly based on the homogenization method. Table【examplifies how different upscaled formulations arise upon different assumptions initially (i.e., on the macroscale) made.

TABLE I. Effective PNP equations: Various different upscaled formulations arise under different assumptions. We abbreviate by "PB" the "Poisson-Boltzmann equations", by "PNP" the "PNP equations", and by "Onsager" the "Onsager reciprocal relations".

\begin{tabular}{|c|c|c|c|c|}
\hline Article & $\mathrm{PB}$ & linearized PNP & PNP & Onsager \\
\hline $\begin{array}{lll}\text { Jackson } & \text { et } & \text { al. } \\
1963,22 & & \\
\end{array}$ & $\begin{array}{lr}\text { - } & \text { given periodic electric } \\
\text { potential or PB equa- } \\
\text { tions } \\
\text { - stationary case } \\
\text { - effective } \\
\text { sion equation, no } \\
\text { homogenization }\end{array}$ & & & \\
\hline $\begin{array}{lll}\text { Gross } & \text { et al. } \\
1968, \underline{23} & \end{array}$ & - no homogenization & & & $\checkmark$ \\
\hline $\begin{array}{lrl}\text { M. } & \text { H. } & \text { Holmes } \\
1990,17 & \end{array}$ & & & $\begin{array}{l}\text { - generalization of Jackson } \\
\text { et al. } 1963 \text { including surface } \\
\text { charge } \\
\text { - homogenization in Slot- } \\
\text { boom variables } \\
\text { - surface charge }\end{array}$ & \\
\hline $\begin{array}{lrr}\begin{array}{l}\text { Moyne } \\
2002, \frac{18}{=},\end{array} & \text { al. } \\
\text { Moyne } & \text { et } \\
2004,24 & & \text { al. }\end{array}$ & & & $\begin{array}{l}\text { - similar to Ray et al. 2011: } \\
\text { Kind of thin double layer } \\
\text { limit (PB in reference cells) } \\
\text { - surface charge }\end{array}$ & \\
\hline $\begin{array}{lll}\text { Looker } & \text { et } & \text { al. } \\
2006,13 & & \end{array}$ & & $\begin{array}{l}\text { - local equilibrium } \\
\text { - local electroneutrality } \\
\text { - fluid flow } \\
\text { - surface charge }\end{array}$ & & $\checkmark$ \\
\hline $\begin{array}{l}\text { Allaire et al. } \\
2010, \frac{12}{2}, \text { based } \\
\text { on Looker et al. } \\
2006,13\end{array}$ & & $\begin{array}{l}\text { - local equilibrium } \\
\text { - bulk electroneutrality } \\
\text { - fluid flow } \\
\text { - surface charge }\end{array}$ & & $\begin{array}{l}-\quad \text { rigorous } \\
\text { with two-scale } \\
\text { convergence }\end{array}$ \\
\hline $\begin{array}{l}\text { Schmuck } 2011, \frac{15}{15} \\
\text { Schmuck } 2012,25\end{array}$ & & & $\begin{array}{l}\text { - local equilibrium } \\
\text { - without surface charge }\end{array}$ & \\
\hline $\begin{array}{lll}\text { Ray et } & \text { al. } \\
2012,26 & & \end{array}$ & & & $\begin{array}{l}\text { - Debye length as homoge- } \\
\text { nization parameter } \\
\text { - without surface charge } \\
\text { - fluid flow }\end{array}$ & \\
\hline
\end{tabular}


New porous medium Poisson-Nernst-Planck equations

Based on the stationary Nernst-Planck equations and a given periodic electric potential, the authors derive an effective diffusion equation for ion densities in 22. In 23, a model for charged membranes separating dilute aqueous salt solutions is studied. The authors give expressions for Onsager's reciprocal relations $\frac{27,28}{2}$ without assuming small Debye-lengths nor a Debye-Hückel linearizaion of the Poisson-Boltzmann equation. Based on ideas from 22, Holmes ${ }^{17}$ performs an asymptotic two-scale expansion for a PNP formulation rewritten in Slotboom variables. This work gives a very interesting approach for nonlinear diffusion in charged polymers. The results seem to be closely related to upscaling of electrostatic potentials $\phi_{0}(y)$ only depending on the micro-scale $y$ (which is related to the use of periodic potentials in 22).

In 18, a macroscopic electrokinetic formulation describing electro-osmotic flow and electrophoretic motion in periodic porous media is obtained by the classical multiple-scale expansion method. They also perform a kind of thin double layer approximation in the reference cells by Poisson-Boltzmann equations. This approximation is well-known and frequently applied in electro-chemistry. ${ }^{29}$ The same authors 24 apply the periodic homogenization theory to upscale the Nernst-Planck equations in the medical and biological context of cartilage.

In 13, the fundamental Onsager reciprocal relations 27,28 together with positive definiteness of corresponding upscaled tensors are derived under the assumption of local thermodynamic equilibrium. Their starting point is a linearized 30 -component electrolyte in a dilute Newtonian fluid (small Reynolds number) flowing through a periodic porous medium. The authors also consider the physically interesting case of surface charge. However, the influence of the surface charge is not obvious at the end. The impact of such charges on the macroscopic level is of major scientific and engineering interest in microfluidics $31-33$ and neurobiology $\mathrm{y}^{4}$.

Very recently, Allaire et al $\stackrel{12}{\underline{12}}$ put the physically relevant derivations from 13 into the rigorous framework of the two-scale convergence. The main purpose is again the verification of Onsager's reciprocal relations as in 13. Derivations of such relations require the assumption of local thermodynamic equilibrium, a linearized PNP system,, 20 and an electroneutrality assumption in the bulk which is physically closely related to a thin double layer approximation.

In 14, the authors perform a singular limit with respect to the dimensionless Debye length. A weighted Debye length, i.e., $\lambda^{\alpha}$, and the use of $\lambda$ as the homogenization parameter has 
New porous medium Poisson-Nernst-Planck equations

the meaning of upscaling the PNP system parallel to taking special ( $\alpha>0$ arbitrary) thin double layer limits of the system. Espeically for $\alpha=1$, this is an interesting problem since the thin double layer approximation is a widely used simplification as already mentioned above.

\section{NOTATION AND PRELIMINARIES}

The following classical exposition recalls central definitions and results from 9-11. For the microscopic variable $y:=\frac{x}{s}$, we obtain the following relation for gradients applied to functions $\psi_{s}(x):=\psi\left(x, \frac{x}{s}\right)$, i.e.,

$$
\nabla \psi_{s}(x)=\nabla_{x} \psi(x, y)+\frac{1}{\epsilon} \nabla_{y} \psi(x, y) .
$$

Homogeneous Neumann problems for Poisson equations for example require the use of the quotient space $W_{\sharp}(Y):=H_{\sharp}^{1}(Y) / \mathbb{R}$. This space of equivalence classes is defined with respect to the relation,

$$
u \simeq v \quad \Leftrightarrow \quad u-v \text { is a constant, } \forall u, v \in H_{\sharp}^{1}(Y) .
$$

For notational brevity, we do not introduce additional notation for an element of this equiva-

lence class. A representative element of this equivalence class can be chosen by the following mean zero condition, that means,

$$
W_{\sharp}(Y):=\left\{u \in H_{\sharp}^{1}(Y) \mid \mathcal{M}_{Y}(u)=0\right\},
$$

where,

$$
\mathcal{M}_{Y}(u):=\frac{1}{|Y|} \int_{Y} u(y) d y
$$

Lemma II.1 The following quantitiy,

$$
\|u\|_{W_{\sharp}}=\|\nabla u\|_{L^{2}(Y)} \quad \forall u, v \in W_{\sharp}(Y),
$$

defines a norm on $W_{\sharp}(Y)$. Moreover, the dual space $\left(W_{\sharp}(Y)\right)^{\prime}$ can be identified by the set,

$$
\left\{F \in\left(H_{\sharp}^{1}(Y)\right) \mid F(c)=0 \quad \forall c \in \mathbb{R}\right\},
$$


New porous medium Poisson-Nernst-Planck equations

with,

$$
\langle F, u\rangle_{\left(W_{\sharp}(Y)\right)^{\prime}, W_{\sharp}(Y)}=\langle F, u\rangle_{\left(H_{\sharp}^{1}(Y)\right)^{\prime}, H_{\sharp}^{1}(Y)} \quad \forall u \in W_{\sharp}(Y) .
$$

Definition II.2 Let $c, C \in \mathbb{R}$ be such that $0<c<C$ and let $D \subset \mathbb{R}^{N}$. We call $N \times N$ matrices $A=\left\{a_{i j}\right\}_{1 \leq i, j \leq N} \in\left(L^{\infty}(U)\right)^{N \times N}$ strongly elliptic, if for any $u \in \mathbb{R}^{N}$ and a.e. in $D$ it holds that,

$$
\begin{gathered}
(A(x) u, u) \geq c|u|^{2}, \\
|A(x) u| \leq C|u| .
\end{gathered}
$$

In our analysis we mainly have to deal with $A=\left\{\delta_{i j}\right\}_{1 \leq i, j \leq N}$ which obvously satisfies the conditions of Definition $\amalg .2$.

Theorem II.3 Let $A$ be a strongly elliptic matrix with $Y$-periodic coefficients and $f \in$ $\left(W_{\sharp}(Y)\right)^{\prime}$. Then the problem,

$$
\left\{\begin{array}{l}
\text { Find } u \in W_{\sharp}(Y) \text { such that } \\
(A \nabla u, \nabla v)_{Y}=(f, v) \quad \forall v \in W_{\sharp}(Y) .
\end{array}\right.
$$

has a unique solution. Moreover,

$$
\|u\|_{W_{\sharp}(Y)} \leq \frac{1}{c}\|f\|_{\left(W_{\sharp}(Y)\right)} .
$$

Remark II.4 Since Theorem II.3 makes a uniqueness statement, we consider in this case the space $W_{\sharp}(Y)$ defined in (5). We apply this convention in the whole article.

We frequently use the following space,

$$
V\left(\Omega_{T}\right):=L^{\infty}(] 0, T\left[; L^{2}(\Omega)\right) \cap L^{2}(] 0, T\left[; H^{1}(\Omega)\right)
$$

\section{A. Review of the classical PNP equations}

Before we come to the main results in this article, we briefly recall basics about the PNP system. In view of computational convenience (block matrix solvers, e.g. 34), notational 
clearity and compactness, and a non-linear (i.e., non-symmetric) extension of the classical Onsager relations, 27,28 which classically only hold in the linearized case, $, 2,13$ motivate us to recall the PNP equations from 15 written by field vectors $\mathbf{u}:=\left[n^{+}, n^{-}, \Phi\right]^{\prime}$ as,

$$
\begin{array}{rlrl}
\mathbf{D}_{t} \mathbf{u}-\Delta_{\mathbb{S}} \mathbf{u}=\mathbf{I}(\mathbf{u}), & \text { for }(\mathbf{t}, \mathbf{x}) \in \boldsymbol{\Omega}_{T}:=\left[\Omega_{T}, \Omega_{T}, \Omega_{T}\right]^{\prime}, \\
\mathbf{u}(0, x)=\mathbf{h}, & \text { in } \boldsymbol{\Omega}:=[\Omega, \Omega, \Omega]^{\prime}, \\
\mathbf{u}=\mathbf{g}_{l}, & \text { on } \boldsymbol{\Gamma}_{T}^{l}:=\left[\Gamma_{T}^{l}, \Gamma_{T}^{l}, \Gamma_{T}^{l}\right]^{\prime}, & \\
\mathbf{u}=\mathbf{g}_{r}, & \text { on } \boldsymbol{\Gamma}_{T}^{r}:=\left[\Gamma_{T}^{l}, \Gamma_{T}^{l}, \Gamma_{T}^{l}\right]^{\prime}, & \\
\left(\mathbb{S}(\mathbf{u}) \nabla_{\mathbf{n}} \mathbf{u}\right)^{i}:=s_{i j}(\mathbf{u}) \nabla_{n}^{j} \mathbf{u}^{j}=0, & \text { on } \boldsymbol{\Gamma}_{T}^{N}:=\partial \boldsymbol{\Omega}_{T} \backslash \boldsymbol{\Gamma}_{T}^{D}, \quad \text { for } i=1,2,3,
\end{array}
$$

where $\mathbf{x}:=[x, x, x]^{\prime}$ with $x \in \Omega \subset \mathbb{R}^{N}$ corresponds to the coordinate field for each component of the field vector $\mathbf{u}$ and $\mathbf{t}:=[t, t, t]^{\prime}$ with $\left.t \in\right] 0, T\left[\right.$ for any $T \in \mathbb{R}_{+}$is the accordingly defined time field. The variables $\mathrm{u}^{1}=n^{+}, \mathrm{u}^{2}=n^{-}$, and $\mathrm{u}^{3}=\Phi$ represent the concentration of positive ions, the density of negative ions, and the induced electric potential, respectively. We further use the convention $\left.\Omega_{T}:=\right] 0, T\left[\times \Omega\right.$. The notation $\Omega_{T}$ accounts for the fact that the components of the field vector $\mathbf{u}$ are defined in different domains of the porous medium later on, i.e., either in the whole domain $\Omega$ or only in the electrolyte phase $\Omega^{s}$. We further denote $\Delta_{\mathbb{S}} \mathbf{u}:=\operatorname{div}(\mathbb{S}(\mathbf{u}) \boldsymbol{\nabla} \mathbf{u})$ with $\mathbb{S}(\mathbf{u}):=\left\{s_{i_{k} j_{l}}(\mathbf{u})\right\}_{\substack{1 \leq i, j \leq d \\ 1 \leq k, l \leq N}}$ for the field indices $1 \leq i, j \leq 3$, the coordinate indices $1 \leq k, l \leq N$ and $s_{i_{k} j_{l}}(\mathbf{u})=s_{i j}(\mathbf{u}) \delta_{k l}$ with $\delta_{k l}$ the Kronecker symbol, $\nabla_{n}:=\mathrm{n} \cdot \nabla$ with $\mathrm{n}$ the normal vector pointing outward of $\Omega, \Gamma_{T}^{D}:=\left[\Gamma_{T}^{D}, \Gamma_{T}^{D}, \Gamma_{T}^{D}\right]^{\prime}$ with $\Gamma_{T}^{D}:=\Gamma_{T}^{l} \cup \Gamma_{T}^{r}$ and $\Gamma_{T}^{N}, \Gamma_{T}^{\iota}$ for $\iota=r, l$ are correspondingly defined, and

$$
\begin{aligned}
& \mathbf{D}_{\mathbf{t}}:= {\left[\begin{array}{lll}
\partial_{t} & 0 & 0 \\
0 & \partial_{t} & 0 \\
0 & 0 & 0
\end{array}\right], \quad\left\{s_{i j}(\mathbf{u})\right\}_{1 \leq i, j \leq 3}:=\left[\begin{array}{ccc}
1 & 0 & n^{+} \\
0 & 1 & -n^{-} \\
0 & 0 & \lambda^{2}
\end{array}\right], \quad \boldsymbol{\nabla}:=\mathbb{I} \nabla:=\left[\begin{array}{ccc}
\nabla & 0 & 0 \\
0 & \nabla & 0 \\
0 & 0 & \nabla
\end{array}\right], } \\
& \operatorname{div}:=\mathbb{I} \operatorname{div}:=\left[\begin{array}{ccc}
\operatorname{div} & 0 & 0 \\
0 & \operatorname{div} & 0 \\
0 & 0 & \operatorname{div}
\end{array}\right], \quad \mathbf{u}:=\left[n^{+}, n^{-}, \Phi\right]^{\prime}, \quad \mathbf{I}(\mathbf{u}):=\left[0,0, n^{+}-n^{-}\right]^{\prime}, \\
& \mathbf{g}_{l}:=\left[n_{l}^{+}, n_{l}^{-}, \phi_{l}\right]^{\prime}, \quad \mathbf{g}_{r}:=\left[n_{r}^{+}, n_{r}^{-}, \phi_{r}\right]^{\prime}, \quad \mathbf{h}:=\left[\mathrm{h}^{1}, \mathrm{~h}^{2}, 0\right]^{\prime} .
\end{aligned}
$$

$\Gamma^{\iota}$ represents the Dirichlet (for $\iota=D$ ) and Neumann (for $\iota=N$ ) boundary surrounding the porous medium $\Omega$, i.e. $\partial \Omega=\Gamma^{D} \cup \Gamma^{N}$. Hence, the first equation (14) 1 is equivalent to the 
New porous medium Poisson-Nernst-Planck equations

following classical system,

$$
\begin{aligned}
\partial_{t} n^{+} & =\operatorname{div}\left(\nabla n^{+}+n^{+} \nabla \Phi\right), \\
\partial_{t} n^{-} & =\operatorname{div}\left(\nabla n^{-}-n^{-} \nabla \Phi\right), \\
-\lambda^{2} \Delta \Phi & =n^{+}-n^{-},
\end{aligned}
$$

which can be interpreted as a gradient flow of the following free energy,

$$
F=U-T S=\int\left(\sum_{i} \mathrm{u}^{i}\left(\log \mathrm{u}^{i}-1\right)+\sum_{i} z_{i} \mathrm{u}^{i} \Phi-\lambda^{2}|\nabla \Phi|^{2}\right) d x
$$

We recall that (17) builds the basis of dilute solution theory which accounts for thermodynamic quantities such as entropy $S$ formed by the first summand in the integral (17). The remaining integrands such as energy density of interactions (second term) and energy density of the electric field (third term) constitute to the internal energy $U$. We note that from the energy (17) we can obtain the chemical potential of the ion densities $\mathrm{u}^{1}=n^{+}$and $\mathrm{u}^{2}=n^{-}$by taking the first variation with respect to $\mathrm{u}^{1}$ and $\mathrm{u}^{2}$, respectively.

An interesting question is whether the minimization of the free energy (17) by a gradient flow also follows the physically relevant path far from thermodynamic equilibrium. In which physical sense does the flow with respect to the Wasserstein distance ${ }^{35-38}$ provide optimality?

\section{THE MICROSCOPIC POROUS MEDIUM FORMULATION AND MAIN RESULTS}

The study in this article relies on the system (14) reformulated for periodic porous media. A scaling parameter $s$ is defined as the ratio between the microscopic length scale $\ell$ and the macroscopic size $L$ of the porous medium, i.e. $s:=\frac{\ell}{L} \ll 1$. It is assumed that $s$ scales the periodicity of the reference cell $Y \subset \mathbb{R}^{N}$ which defines the micro-geometry. In this reference cell, we denote the fluid (liquid) region by $Y^{s} \subset Y$ such that its complement is the solid phase. After periodically covering the domain $\Omega$ by such cells $Y$, we denote the resulting macroscopic domain of the periodic union of the subsets $Y^{s}$ by $\Omega^{s}$ and its complement by $B^{s}:=\Omega \backslash \Omega^{s}$. Hence, the perforated domain $B^{s}$ represents the solid phase and $\Omega^{s}$ the liquid phase, see Figure 1. 
New porous medium Poisson-Nernst-Planck equations

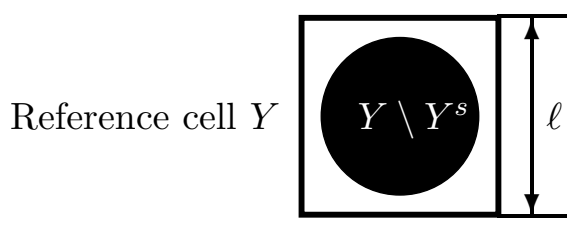

Periodic covering by scaled cells $Y$

\begin{tabular}{|c|c|c|c|c|c|c|}
\hline$B^{5}$ & $B^{s}$ & $B^{s}$ & $B^{5}$ & $B^{5}$ & $B^{s}$ & \multirow{3}{*}{$(s \rightarrow 0)$} \\
\hline$B^{s}$ & $B^{s}$ & $B^{5}$ & $B^{s}$ & $B^{s}$ & $B^{s}$ & \\
\hline$\overline{B^{5}}$ & $B^{s}$ & $B^{5}$ & $\overline{B^{5}}$ & $\widehat{B^{5}}$ & $B^{s}$ & \\
\hline$B^{s}$ & $B$ & $B^{5}$ & $B^{5}$ & $B^{5}$ & $B^{\circ}$ & \\
\hline$B^{5}$ & $B^{s}$ & $B^{5}$ & $B^{5}$ & $B^{5}$ & $B^{s}$ & $\Phi$ \\
\hline
\end{tabular}

Homogenous approximation

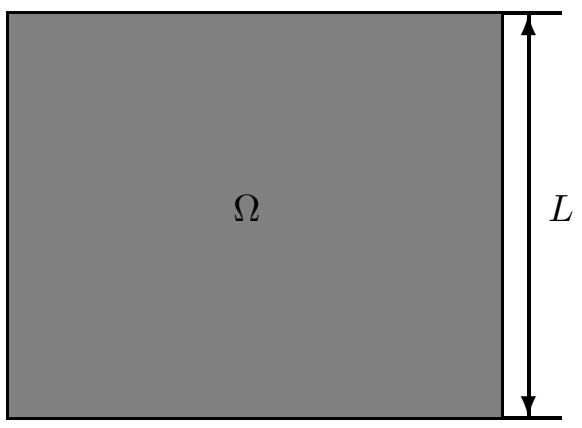

FIG. 1. Left: Strongly heterogeneous material as a periodic covering of reference cells $Y:=[0, \ell]^{N}$.

Top, middle: The reference cell $Y$ represents a characteristic mean pore geometry. Right: The "homogenization limit" $s:=\frac{\ell}{L} \rightarrow 0$ stands here for the leading order approximation of nonstandard two-scale expansions.

Under these considerations, the material tensor $\mathbb{S}$ from $(14)_{2}$ depends now on $s$ too, i.e.,

$$
\left\{s_{i_{k} j_{l}}^{s}\left(\mathbf{x}, \mathbf{u}_{s}\right)\right\}_{1 \leq i, j \leq 3}:=\left[\begin{array}{ccc}
1 & 0 & n_{s}^{+} \\
0 & 1 & -n_{s}^{-} \\
0 & 0 & \epsilon(x / s)
\end{array}\right] \delta_{k l}, \quad \text { for } 1 \leq k, l \leq N
$$

where $\epsilon(x):=\lambda^{2} \chi_{\Omega^{s}}(x)+\alpha \chi_{B^{s}}(x)$ with the classical dimensionless Debye length $\lambda:=\frac{\lambda_{D}}{L}$ of the PNP system (16),$\alpha=\frac{\epsilon_{m}}{\epsilon_{f}}$ is the dimensionless dielectric permittivity where $\epsilon_{m}$ and $\epsilon_{f}$ are the dielectric permittivities of the solid and liquid phase, respectively. In (18) one recognizes that also the physical quantities like concentrations $n_{s}^{+}, n_{s}^{-}$and electric potential depend on the scaling parameter $s$. Hence, the problem (14) reads now in the periodic 
New porous medium Poisson-Nernst-Planck equations

setting as follows,

$$
\begin{aligned}
\mathbf{D}_{t} \mathbf{u}_{s}-\operatorname{div}\left(\mathbb{S}^{s}\left(\mathbf{u}_{s}\right) \boldsymbol{\nabla} \mathbf{u}_{s}\right)=\mathbf{I}\left(\mathbf{u}_{s}\right), & \text { in } \boldsymbol{\Omega}_{T}^{s}:=\left[\Omega_{T}^{s}, \Omega_{T}^{s}, \Omega_{T}\right]^{\prime}, \\
\mathbf{u}_{s}(0, x)=\mathbf{h}_{s}, & \text { in } \boldsymbol{\Omega}^{s}:=\left[\Omega^{s}, \Omega^{s}, \Omega\right]^{\prime}, \\
\mathbb{S}^{s}\left(\mathbf{u}_{s}\right) \boldsymbol{\nabla}_{\mathbf{n}} \mathbf{u}_{s}=\mathbf{0}, & \text { on } \boldsymbol{\Gamma}_{T}^{N}, \\
\mathbf{u}_{s}=\mathbf{g}_{\iota}, & \text { on } \boldsymbol{\Gamma}_{T}^{\iota}, \quad \iota=l, r, \\
\left(\mathbb{S}^{s}\left(\mathbf{u}_{s}\right) \boldsymbol{\nabla}_{\mathbf{n}} \mathbf{u}_{s}\right) \mathbf{f}_{i}=0, & \text { on } I_{T}^{s}, \quad \text { for } i=1,2, \\
\left.\left(\mathbb{S}^{s}\left(\mathbf{u}_{s}\right) \boldsymbol{\nabla}_{\mathbf{n}} \mathbf{u}_{s}\right) \mathbf{f}_{3}\right|_{\Omega_{T}^{s}}=\left.\left(\mathbb{S}^{s}\left(\mathbf{u}_{s}\right) \boldsymbol{\nabla}_{\mathbf{n}} \mathbf{u}_{s}\right) \mathbf{f}_{3}\right|_{B_{T}^{s},} & \text { on } I_{T}^{s}, \\
\left.u_{s}^{3}\right|_{\Omega_{T}^{s}}=\left.u_{s}^{3}\right|_{B_{T}^{s}}, & \text { on } I_{T}^{s},
\end{aligned}
$$

where $\mathbf{f}_{i}:=\left[\delta_{i 1}, \delta_{i 2}, \delta_{i 3}\right]^{\prime}$ for $i=1,2,3$ and $\delta_{i j}$ is the Kronecker delta and $I_{T}^{s}:=\partial \Omega_{T}^{s} \backslash$ $\left\{\Gamma_{T}^{D} \cup \Gamma_{T}^{N}\right\}$ the solid-electrolyte interface. From (19) it follows that the flux with respect to $\mathbf{u}$ is in general not differentiable. This motivates to study (19) in the sense of weak solutions. Moreover, the main difficulty and difference of this work is the nonlinear structure which prevents the material tensor $\mathbb{S}^{s}\left(\mathbf{u}_{s}\right)$ to be a strongly elliptic operator. For convenience, we rewrite (19) for $\left[n_{s}^{+}, n_{s}^{-}, \Phi_{s}\right]=\left[\mathrm{u}_{s}^{1}, \mathrm{u}_{s}^{2}, \mathrm{u}_{s}^{3}\right]$ in the classical form here, that is,

$$
\begin{aligned}
\partial_{t} n_{s}^{+}=\operatorname{div}\left(\nabla n_{s}^{+}+n_{s}^{+} \nabla \Phi_{s}\right) & \text { in } \Omega_{T}^{s}, \\
\partial_{t} n_{s}^{-}=\operatorname{div}\left(\nabla n_{s}^{-}-n_{s}^{-} \nabla \Phi_{s}\right) & \text { in } \Omega_{T}^{s}, \\
-\operatorname{div}\left(\epsilon(x / s) \nabla \Phi_{s}\right)=n_{s}^{+}-n_{s}^{-} & \text {in } \Omega_{T},
\end{aligned}
$$

where the corresponding ionic fluxes are defined by,

$$
\begin{aligned}
& \mathrm{j}_{s}^{+}:=\nabla n_{s}^{+}+n_{s}^{+} \nabla \Phi_{s}, \\
& \mathrm{j}_{s}^{-}:=\nabla n_{s}^{-}-n_{s}^{-} \nabla \Phi_{s},
\end{aligned}
$$

and the boundary and initial conditions are,

$$
\begin{aligned}
n_{s}^{+}=\mathrm{h}_{s}^{1} \quad n_{s}^{-}=\mathrm{h}_{s}^{2} & \text { in } \Omega^{s}, \\
n_{s}^{+}=g_{\iota} \quad n_{s}^{-}=g_{\iota} & \text { on } \Gamma_{T}^{\iota}, \\
\mathrm{j}_{s}^{+} \cdot \mathrm{n}=0 \quad \mathrm{j}_{s}^{-} \cdot \mathrm{n}=0 & \text { on } \Gamma_{T}^{N},
\end{aligned}
$$

where $\iota=l, r$. Our main result relies on the assumption of local thermodynamic equilibrium, which is widely used and generally accepted. $25,33,39,40$ 
New porous medium Poisson-Nernst-Planck equations

Definition III.1 (Local thermodynamic equilibrium) We say that the reference cells $Y$ are in local thermodynamic equilibrium if and only if for each $x / s=y$ of the same reference cell $Y$ it holds that

$$
\mu_{0}^{r}:=\log \mathrm{u}^{r}(t, x)+z_{r} \mathrm{u}^{3}(t, x)=\text { const. }
$$

where $\mu_{0}^{r}$ denotes a constant value of the chemical potential of positive $(r=1)$ and negative $(r=2)$ ion densities. Hence, the locally constant potential $\mu_{0}^{r}$ only assumes different values in different reference cells.

In order to simplify the subsequent derivations, we make the following

Assumption (ABC): (Academic Boundary Conditions) We assume for the ion densities $\mathrm{u}_{s}^{r}$ for $r=1,2$ homogeneous Dirichlet boundary conditions on $\partial \Omega$ and no-flux boundary conditions on the solid-electrolyte interface $I^{s}:=\partial \Omega^{s} \backslash\left\{\Gamma^{D} \cup \Gamma^{N}\right\}$ inside the porous medium $\Omega$. For the electric potential $\mathrm{u}_{s}^{3}=\Phi_{s}$, we assume homogeneous Neumann boundary conditions on the boundary of the porous medium, i.e., $\partial \Omega$, and continuity, i.e., $\left.\Phi\right|_{\Omega^{s}}=\left.\Phi\right|_{B^{s}}$, at the solid-electrolyte interface $I^{s}:=\partial \Omega^{s} \backslash\left\{\Gamma^{D} \cup \Gamma^{N}\right\}$ as well as continuity of the corresponding fluxes, i.e., $\left.\epsilon(x / s) \nabla_{n} \Phi_{s}\right|_{\Omega^{s}}=\left.\epsilon(x / s) \nabla_{n} \Phi_{s}\right|_{B^{s}}$ on $I^{s}$.

These considerations allow us to state our main result which consists of passing to the limit $s \rightarrow 0$. This limit has the physical meaning of homogeneously mixing the solid and the liquid phase in the porous material under constant volume fractions, see Figure 1, Such a homogeneous description of a porous medium is a good approximation if the medium is very heterogeneous, i.e., $s=\frac{\ell}{L}$ is very small.

Theorem III.2 We assume that the reference cells $Y$ are in local thermodynamic equilibrium. The boundary conditions satisfy the Assumption (ABC). Then, the solution $\mathbf{u}_{s}(\mathbf{t}, \mathbf{x}):=\left[\mathrm{u}_{s}^{1}(t, x), \mathrm{u}_{s}^{2}(t, x), \mathrm{u}_{s}^{3}(t, x)\right]$ of problem (19) admits the following formal asymptotic expansions,

$$
\begin{aligned}
& \mathrm{u}_{s}^{r}=\mathrm{u}_{0}^{r}-s \sum_{k=1}^{N} \xi^{r_{k}}(t, x, x / s) \frac{\partial \mathrm{u}_{0}^{3}}{\partial x_{k}}+s^{2} \sum_{k, l=1}^{N} \zeta^{r_{k l}}(t, x, x / s) \mathrm{u}_{0}^{r}+\ldots \quad \text { for } r=1,2, \\
& \mathrm{u}_{s}^{3}=\mathrm{u}_{0}^{3}-s \sum_{k=1}^{N} \xi^{3{ }_{k}}(x / s) \frac{\partial \mathrm{u}_{0}^{3}}{\partial x_{k}}+s^{2} \sum_{k, l=1}^{N} \zeta^{3_{k l}}(x / s) \frac{\partial^{2} \mathrm{u}_{0}^{3}}{\partial_{x_{k}} \partial_{x_{l}}}+\ldots,
\end{aligned}
$$


New porous medium Poisson-Nernst-Planck equations

where $\xi^{r_{k}}(\cdot, \cdot, y) \in V\left(\Omega_{T}, W_{\sharp}\left(Y^{s}\right)\right)$ solves $45, \xi^{3 k}(y) \in W_{\sharp}(Y)$ solves 4 44), $\zeta^{r_{k l}}(\cdot, \cdot, y) \in$ $V\left(\Omega_{T}, W_{\sharp}\left(Y^{s}\right)\right)$ solves (78), $\zeta^{3 k l}(y) \in W_{\sharp}(Y)$ solves (73), and $\mathbf{u}_{0}$ is a solution of the following upscaled system,

$$
\begin{cases}p \partial_{t} \mathrm{u}_{0}^{r}-p \Delta \mathrm{u}_{0}^{r}+\operatorname{div}\left(\mathbb{D}^{r}(t, x) \nabla \mathrm{u}_{0}^{3}\right)-\operatorname{div}\left(z_{r} \mathrm{u}_{0}^{r} \mathbb{M} \nabla \mathrm{u}_{0}^{3}\right)=0 & \text { in } \Omega_{T}, \\ -\operatorname{div}\left(\boldsymbol{\epsilon}^{0} \nabla \mathrm{u}_{0}^{3}\right)=p\left(\mathrm{u}_{0}^{1}-\mathrm{u}_{0}^{2}\right) & \text { in } \Omega_{T},\end{cases}
$$

where $p:=\left|Y^{s}\right| /|Y|$ is the porosity. The tensor $\mathbb{D}^{r}(t, x):=\left\{\mathrm{D}_{k l}^{r}(t, x)\right\}_{1 \leq k, l \leq N}$ related to diffusion, the tensor $\mathbb{M}:=\left\{\mathrm{M}_{k l}\right\}_{1 \leq k, l \leq N}$ related to electro-convection, and the effective permeability tensor $\boldsymbol{\epsilon}^{0}:=\left\{\epsilon_{k l}^{0}\right\}_{1 \leq k . l \leq N}$ are defined by,

$$
\begin{aligned}
\mathrm{D}_{i k}^{r}(t, x) & :=\frac{1}{|Y|} \int_{Y^{s}} \sum_{j=1}^{N}\left\{\delta_{i j} \partial_{y_{j}} \xi^{r_{k}}(t, x, y)\right\} d y \\
\mathrm{M}_{i k} & :=\frac{1}{|Y|} \int_{Y^{s}} \sum_{j=1}^{N}\left\{\delta_{i k}-\delta_{i j} \partial_{y_{j}} \xi^{3_{k}}(y)\right\} d y, \\
\epsilon_{i k}^{0}: & =\frac{1}{|Y|} \int_{Y} \sum_{j=1}^{N} \epsilon(y)\left(\delta_{i k}-\delta_{i j} \partial_{y_{j}} \xi^{3_{k}}(y)\right) d y .
\end{aligned}
$$

The second order correctors $\zeta^{r k l}$ only exist for positive densities $\mathrm{u}_{0}^{r}$.

We note that for the classical asymptotic two-scale expansions ${ }^{9}, 10$ an upscaling is performed in 25 where also error erstimates between the microscopic periodic formulation and the upscaled equations are derived.

Remark III.3 (1) Theorem III.2 is an extension of the two-scale convergence results in 15 by the non-classical asymptotic expansion (24). We note that the expansions (24) are only formal because convergence of such series is a priori not guaranteed and possible boundary layers are neglected.

(2) The effect of the upscaling in the above theorem can be best seen in the change of the material tensor (18), which reads for the new system (25) as follows

$$
\left\{s_{i_{k} j_{l}}^{s}\left(\mathbf{x}, \mathbf{u}_{0}\right)\right\}_{1 \leq i, j \leq 3}:=\left[\begin{array}{ccc}
p \delta_{k l} & 0 & -\mathrm{D}_{k l}^{1}+z_{r} \mathrm{u}_{0}^{1} \mathrm{M}_{k l} \\
0 & p \delta_{k l} & -\mathrm{D}_{k l}^{1}-z_{r} \mathrm{u}_{0}^{2} \mathrm{M}_{k l} \\
0 & 0 & \epsilon_{k l}^{0}(x / s)
\end{array}\right], \quad \text { for } 1 \leq k, l \leq N .
$$

A comparison of (27) with (18) clearly motivates the use of the term "material tensor" in the context of porous or composite media. 
New porous medium Poisson-Nernst-Planck equations

(3) The effective material tensor (27) can also be considered as a generalized effective, concentration dependent conductivity tensor (as in heat/diffusion equations).

We note that the effective macroscopic material tensor (27) reveals that one can controle and enhance material transport by adjusting the contrast in the electric permittivities between the electrolyte and the porous medium. The different upscaling ideas as well as different physical and mathematical assumptions play an important role for an improved understanding of how different microscopic material properties such as pore geometries, electric permittivities, charge numbers of the ions, and surface charges of the porous medium influence the transport characteristics on the macroscale.

Our last result guarantees the existence and uniqueness of solutions for the porous media PNP equations (25). In order to keep the presentation clear and to prevent a technical derivation, we restrict our considerations to purely academic boundary conditions in the next

Lemma III.4 Let $\Omega \subset \mathbb{R}^{N}$ be a domain with smooth boundary $\partial \Omega$ and $T>0$ small enough. The boundary conditions satisfy Assumption (ABC). Then, the coupled system of equations,

$$
\begin{cases}p \partial_{t} \mathrm{u}^{r}-p \Delta \mathrm{u}^{r}+\operatorname{div}\left(\mathbb{D}^{r}(t, x) \nabla \mathrm{u}^{3}\right)-\operatorname{div}\left(z_{r} \mathrm{u}_{0}^{r} \mathbb{M} \nabla \mathrm{u}^{3}\right)=0 & \text { in } \Omega_{T}, \\ -\operatorname{div}\left(\boldsymbol{\epsilon}^{0} \nabla \mathrm{u}^{3}\right)=p\left(\mathrm{u}^{1}-\mathrm{u}^{2}\right) & \text { in } \Omega_{T},\end{cases}
$$

together with the following initial and boundary conditions,

$$
\begin{array}{rlrl}
\mathrm{u}^{r}(0, x) & \in L^{2}(\Omega) \cap H_{0}^{1}(\Omega) & \text { for } r=1,2, \\
\mathrm{u}^{r} & =0 & \text { on } \partial \Omega \times] 0, T[ & \text { for } r=1,2, \\
\nabla_{n} \mathrm{u}^{3} & =0 & \text { on } \partial \Omega \times] 0, T[, &
\end{array}
$$

has unique weak solutions $\mathrm{u}^{r} \in V\left(\Omega_{T}\right):=L^{\infty}(] 0, T\left[; L^{2}(\Omega)\right) \cap L^{2}(] 0, T\left[; H_{0}^{1}(\Omega)\right)$ and $\mathrm{u}^{3} \in$ $L^{\infty}(] 0, T\left[; H^{2}(\Omega)\right)$. That means, $\mathrm{u}^{r}(t, x), r=1,2$, and $\mathrm{u}^{3}(t, x)$ solve

$$
\left\{\begin{array}{cc}
p \frac{d}{d t}\left(\mathrm{u}^{r}, \varphi^{r}\right)+p\left(\nabla \mathrm{u}^{r}, \nabla \varphi^{r}\right)-\left(\mathbb{D}^{r}(t, x) \nabla \mathrm{u}^{3}, \nabla \varphi^{r}\right) & \\
+\left(z_{r} \mathrm{u}^{r} \mathbb{M} \nabla \mathrm{u}^{3}, \varphi^{r}\right)=0 & \forall \varphi^{r} \in H_{0}^{1}(\Omega), \\
\left(\epsilon^{0} \nabla \mathrm{u}^{3}, \nabla \varphi^{3}\right)=p\left(\mathrm{u}^{1}-\mathrm{u}^{2}, \varphi^{3}\right) & \forall \varphi^{3} \in \bar{H}^{1}(\Omega),
\end{array}\right.
$$

where $\bar{H}^{1}(\Omega):=\left\{u \in H^{1}(\Omega) \mid \int_{\Omega} u d x=0\right\}$.

From a rigorous point of view, Lemma III.4 finally guarantees that the second order terms in the asymptotic expansion (24) are locally well-defined. 
New porous medium Poisson-Nernst-Planck equations

\section{PROOF OF THEOREM III.2}

We first rewrite the second term in equation $(19)_{1}$ with help of the property (3) in the following way

$$
\begin{aligned}
\operatorname{div}\left(\mathbb{S}^{s}\left(\boldsymbol{\psi}_{s}, \mathbf{x}\right) \boldsymbol{\nabla} \boldsymbol{\psi}_{s}\right) & =s^{-2} \boldsymbol{\operatorname { d i v }}_{y}\left(\mathbb{S}\left(\boldsymbol{\psi}_{s}, \mathbf{y}\right) \boldsymbol{\nabla}_{y} \boldsymbol{\psi}_{s}\right) \\
& +s^{-1}\left\{\operatorname{div}_{x}\left(\mathbb{S}\left(\boldsymbol{\psi}_{s}, \mathbf{y}\right) \boldsymbol{\nabla}_{y} \boldsymbol{\psi}_{s}\right)+\operatorname{div}_{y}\left(\mathbb{S}\left(\boldsymbol{\psi}_{s}, \mathbf{y}\right) \boldsymbol{\nabla}_{x} \boldsymbol{\psi}_{s}\right)\right\} \\
& +\operatorname{div}_{x}\left(\mathbb{S}\left(\boldsymbol{\psi}_{s}, \mathbf{y}\right) \boldsymbol{\nabla}_{x}\right) \\
& :=\left[s^{-2} \mathcal{S}_{0}+s^{-1} \mathcal{S}_{1}+\mathcal{S}_{2}\right] \boldsymbol{\psi}\left(\mathbf{x}, \frac{\mathbf{x}}{s}\right)
\end{aligned}
$$

where $\boldsymbol{\psi}_{s}(\mathbf{x})$ is an arbitrary function as in (3) . For the subsequent considerations we apply the following notation,

$$
\begin{aligned}
{\left[\mathcal{S}_{2}\right]^{N} \boldsymbol{\psi}_{s} } & :=\left[\operatorname{div}\left(\mathbb{S}\left(\boldsymbol{\psi}_{s}, \mathbf{y}\right) \boldsymbol{\nabla}_{y} \boldsymbol{\psi}_{s}\right)\right]^{N}:=\left[\operatorname{div}\left(\mathbb{S}\left(\boldsymbol{\psi}_{s}, \mathbf{y}\right) \boldsymbol{\nabla}_{y} \boldsymbol{\psi}_{s}\right)\right]^{N} \\
& :=\left\{\operatorname{div}_{x}\left(\mathrm{~s}_{i j}(\boldsymbol{\psi}, \mathbf{y}) \nabla_{x} \psi_{s}^{j}\right) \mathbf{f}_{i}\right\}_{(i=1, j=3) \cup(i=2, j=3)} \\
& =\operatorname{div}_{x}\left(\psi_{s}^{1} \nabla_{x} \psi_{s}^{3}\right) \mathbf{f}_{1}-\operatorname{div}_{x}\left(\psi_{s}^{2} \nabla_{x} \psi_{s}^{3}\right) \mathbf{f}_{2} .
\end{aligned}
$$

Hence, the operator $[\cdot]^{N}$ represents a restriction to nonlinear components given by the indices $(i=1, j=3)$ and $(i=2, j=3)$.

We make now the formal Ansatz of the asymptotic expansion

$$
\mathbf{u}_{s}(\mathbf{t}, \mathbf{x}) \approx \mathbf{u}_{0}(\mathbf{t}, \mathbf{x}, \mathbf{x} / s)+s \mathbf{u}_{1}(\mathbf{t}, \mathbf{x}, \mathbf{x} / s)+s^{2} \mathbf{u}_{2}(\mathbf{t}, \mathbf{x}, \mathbf{x} / s)+\ldots
$$

with $\mathbf{u}_{i}(\mathbf{t}, \mathbf{x}, \mathbf{y})$ for $i=0,1,2, \ldots$ such that

$$
\begin{cases}\mathbf{u}_{i}(\mathbf{t}, \mathbf{x}, \mathbf{y}) & \text { is defined for }(\mathbf{t}, \mathbf{x}) \in \boldsymbol{\Omega}_{T}^{s} \text { and } \mathbf{y} \in \mathbf{Y}:=\left[Y^{s}, Y^{s}, Y\right]^{\prime}, \\ \mathbf{u}_{i}(\cdot, \cdot, \mathbf{y}) & \text { is } \mathbf{Y} \text {-periodic. }\end{cases}
$$

The above Ansatz is formal because there is no guarantee that the series (33) is finite.

(1) Problem for terms of order $\mathcal{O}\left(s^{-2}\right)$ : After inserting (33) into (19) 1 , using (31) and (32) gives a sequence of problems by equating terms with equal power in $s$, that means,

$$
\mathcal{O}\left(s^{-2}\right): \quad\left\{\begin{array}{l}
-\mathcal{S}\left(\mathbf{u}_{0}, \mathbf{y}\right) \mathbf{u}_{0}=0 \\
\mathbf{u}_{0}(\cdot, \mathbf{y}) \mathbf{Y} \text {-periodic } .
\end{array} \quad \text { in } \mathbf{Y}\right.
$$

If we use definition (31), then we can rewrite (35) 1 as the following equation,

$$
-\operatorname{div}\left(\mathbb{S}\left(\mathbf{u}_{0}, \mathbf{y}\right) \nabla_{y} \mathbf{u}_{0}\right)=\mathbf{0} \quad \text { in } \mathbf{Y}
$$


New porous medium Poisson-Nernst-Planck equations

wich is equivalent to the system,

$$
\mathcal{O}\left(s^{-2}\right): \quad \begin{cases}-\Delta_{y} \mathrm{u}_{0}^{1}-\operatorname{div}_{y}\left(\mathrm{u}_{0}^{1} \nabla_{y} \mathrm{u}_{0}^{3}\right)=0 & \text { in } Y^{s}, \\ -\Delta_{y} \mathrm{u}_{0}^{2}+\operatorname{div}_{y}\left(\mathrm{u}_{0}^{2} \nabla_{y} \mathrm{u}_{0}^{3}\right)=0 & \text { in } Y^{s} \\ -\operatorname{div}_{y}\left(\kappa(y) \nabla_{y} \mathrm{u}_{0}^{3}\right)=0 & \text { in } Y .\end{cases}
$$

One recognizes immediately that solvability must be first established for equation (37) 3 . This is immediately achieved by Theorem II.3. Moreover, this theorem implies that $\mathrm{u}_{0}^{3}(x, y)$ is invariant (constant) in $y \in Y$ as a solution of $(\underline{37})_{3}$, i.e.,

$$
\mathrm{u}_{0}^{3}(t, x, y)=\mathrm{u}_{0}^{3}(t, x)
$$

Using invariance (38) in equations $(\underline{37})_{1}$ and $(\underline{37})_{2}$ implies with Theorem II.3 the additional invariances

$$
\mathrm{u}_{0}^{1}(t, x, y)=\mathrm{u}_{0}^{1}(t, x) \quad \text { and } \quad \mathrm{u}_{0}^{2}(t, x, y)=\mathrm{u}_{0}^{2}(t, x)
$$

Let us go over to the next problem in the sequence of equal power in $s$.

(2) Problem for terms of order $\mathcal{O}\left(s^{-1}\right)$ : (Reference cell problems) The second problem has the form,

$$
\mathcal{O}\left(s^{-1}\right):\left\{\begin{array}{l}
-\mathcal{S}_{0}\left(\mathbf{u}_{0}, \mathbf{y}\right) \mathbf{u}_{1}=\left[\mathcal{S}_{0}\left(\mathbf{u}_{1}, \mathbf{y}\right)\right]^{N} \mathbf{u}_{0}+\mathcal{S}_{1}\left(\mathbf{u}_{0}, \mathbf{u}_{0}, \mathbf{y}\right) \mathbf{u}_{0} \quad \text { in } Y \\
\mathbf{u}_{1}(\cdot, \mathbf{u}) Y \text {-periodic }
\end{array}\right.
$$

Let us write (40) in a more intuitive form by its single components, i.e.,

$$
\mathcal{O}\left(s^{-1}\right):\left\{\begin{aligned}
-\Delta_{y} \mathrm{u}_{1}^{1}- & \operatorname{div}_{y}\left(\mathrm{u}_{0}^{1} \nabla_{y} \mathrm{u}_{1}^{3}\right)=\operatorname{div}_{y}\left(\mathrm{u}_{1}^{1} \nabla_{y} \mathrm{u}_{0}^{3}\right)+\operatorname{div}_{x} \nabla_{y} \mathrm{u}_{0}^{1} \\
+\operatorname{div}_{x}\left(\mathrm{u}_{0}^{1} \nabla_{y} \mathrm{u}_{0}^{3}\right)+\operatorname{div}_{y} \nabla_{x} \mathrm{u}_{0}^{1}+\operatorname{div}_{y}\left(\mathrm{u}_{0}^{1} \nabla_{x} \mathrm{u}_{0}^{3}\right) & \text { in } Y^{s}, \\
-\Delta_{y} \mathrm{u}_{1}^{2}-\operatorname{div}_{y}\left(\mathrm{u}_{0}^{2} \nabla_{y} \mathrm{u}_{1}^{3}\right)=-\operatorname{div}_{y}\left(\mathrm{u}_{1}^{2} \nabla_{y} \mathrm{u}_{0}^{3}\right)+\operatorname{div}_{x} \nabla_{y} \mathrm{u}_{0}^{2} & \\
-\operatorname{div}_{x}\left(\mathrm{u}_{0}^{2} \nabla_{y} \mathrm{u}_{0}^{3}\right)+\operatorname{div}_{y} \nabla_{x} \mathrm{u}_{0}^{2}-\operatorname{div}_{y}\left(\mathrm{u}_{0}^{2} \nabla_{x} \mathrm{u}_{0}^{3}\right) & \text { in } Y^{s}, \\
-\operatorname{div}_{y}\left(\kappa(y) \nabla_{y} \mathrm{u}_{1}^{3}\right)=\operatorname{div}_{x}\left(\kappa(y) \nabla_{y} \mathrm{u}_{0}^{3}\right) & \\
+\operatorname{div}_{y}\left(\kappa(y) \nabla_{x} \mathrm{u}_{0}^{3}\right) & \text { in } Y .
\end{aligned}\right.
$$

The system (41) is a linear, elliptic second order partial differential equation. Hence solvability of (41) follows immediately with Lax-Milgram's Theorem by starting with problem 
New porous medium Poisson-Nernst-Planck equations

(41) 3 . The fact that $\mathbf{u}_{0}$ is independent of $\mathbf{y}$ together with the linearity of (41) and that $\mathcal{S}_{0}$ only contains derivatives in $\mathbf{y}$, motivates to make the following Ansatz for $\mathbf{u}_{1}(t, \mathbf{x}, \mathbf{y})$, that means,

$$
\begin{gathered}
\mathrm{u}_{1}^{r}(t, x, y)=-\sum_{j=1}^{N} \xi^{r_{j}}(t, x, y) \partial_{x_{j}} \mathrm{u}_{0}^{3}(t, x) \quad \text { for } r=1,2 \\
\mathrm{u}_{1}^{3}(t, x, y)=-\sum_{j=1}^{N} \xi^{3_{j}}(y) \partial_{x_{j}} \mathrm{u}_{0}^{3}(t, x) .
\end{gathered}
$$

We use now (42) and the independence of $\mathbf{u}_{0}$ of $\mathbf{y}$ in order to rewrite (41) as a problem for $\xi^{r_{j}}$ for $r=1,2$ and $1 \leq j \leq N$ as follows,

$$
\left\{\begin{array}{cc}
-\Delta_{y} \xi^{r_{j}}(t, x, y) \partial_{x_{j}} \mathrm{u}_{0}^{3}-\sum_{i=1}^{N} \partial_{y_{i}}\left(\delta_{i j}\left(\partial_{x_{j}} \mathrm{u}_{0}^{r}+z_{r} \mathrm{u}_{0}^{r} \partial_{x_{j}} \mathrm{u}_{0}^{3}\right)\right) & \\
=-\operatorname{div}_{y}\left(z_{r} \mathrm{u}_{0}^{r} \nabla_{y} \xi^{3_{j}}(y)\right) \partial_{x_{j}} \mathrm{u}_{0}^{3} & \text { in } \Omega^{s} \times Y^{s}, \\
-\operatorname{div}_{y}\left(\kappa(y) \nabla_{y} \xi^{3 j}(y)\right)+\sum_{i=1}^{N} \partial_{y_{i}}\left(\kappa(y) \delta_{i j}\right)=0 & \text { in } \Omega \times Y .
\end{array}\right.
$$

We point out that under local thermodynamic equilibrium, that means, in each reference cell we have due to the induced separation of scales by the limit $\epsilon \rightarrow 0$,

$$
\partial_{x_{j}} u_{0}^{r}=-z_{r} u_{0}^{r} \partial_{x_{j}} u_{0}^{3}
$$

see Definition III.1. Hence, the term with the summation on the left-hand side in (43) 1 disappears. System (43) defines the reference cell problems for the porous media corrector functions $\xi^{r_{j}}$ for $r=1,2$ and $1 \leq j \leq N$. Such correctors finally define the effective tensors (26).

Remark IV.1 We point out that the Ansatz (42) is an extension from linear homogenization theory and is canonically chosen to account for the problem's coupled and nonlinear structure. The interpretation of the Ansatz (42) 1 is that oscillations in the microscopic variable of the electrostatic potential dominate the oscillations of the concentration variables.

Lemma IV.2 Let $\mathrm{u}_{0}^{r} \in V\left(\Omega_{T}\right)$. There exists a unique solution $\xi^{3_{j}}(y) \in W_{\sharp}(Y)$ for each $1 \leq j \leq N$ of problem $(43)_{2}$, i.e.,

$$
\left\{\begin{array}{l}
\text { Find } \xi^{3_{j}} \in W_{\sharp}(Y) \text { such that } \\
\mathrm{a}_{1}^{3}\left(\xi^{3_{j}}, w\right)=\mathrm{F}_{1}^{3}(w):=\sum_{i=1}^{N}\left(\kappa(y) \delta_{i j}, \partial_{y_{i}} w\right)_{Y} \quad \forall w \in W_{\sharp}(Y),
\end{array}\right.
$$


New porous medium Poisson-Nernst-Planck equations

where $\mathrm{a}_{1}^{3}\left(\xi^{3_{j}}, w\right):=\left(\kappa(y) \nabla_{y} \xi^{3_{j}}, \nabla_{y} w\right)_{Y}$. With $\xi^{3_{l}}(y) \in W_{\sharp}(Y)$ for $1 \leq l \leq N$ also the existence and uniqueness of a solution $\xi^{r_{j}}(t, x, y) \in V\left(\Omega_{T}, W_{\sharp}\left(Y^{s}\right)\right)$ of problem (43) follows, that means,

$$
\left\{\begin{array}{c}
\text { For each } 1 \leq j \leq N \text { find } \xi^{r_{j}}(t, x, y) \in V\left(\Omega_{T}, W_{\sharp}\left(Y^{s}\right)\right) \text { such that } \\
\mathrm{a}_{1}^{r}\left(\xi^{r_{j}}, w^{r}\right)=\mathrm{F}_{1}^{r_{j}}\left(w^{r}\right) \quad \forall w^{r} \in L^{2}\left(\Omega ; W_{\sharp}\left(Y^{s}\right)\right),
\end{array}\right.
$$

where for all $w^{r}=\phi^{r}(y) \psi^{r}(x) \in H_{0}^{1}\left(\Omega_{T}, W_{\sharp}(Y)\right)$ we define,

$$
\begin{aligned}
\mathrm{a}_{1}^{r}\left(\xi^{r_{j}}, w^{r}\right) & :=\left(\left(\nabla_{y} \xi^{r_{j}}(t, x, y), \nabla_{y} \phi^{r}\right)_{Y^{s}}, \psi^{r}\right)_{\Omega}, \\
\mathrm{F}_{1}^{r_{j}}\left(w^{r}\right) & :=-\left(\left(z_{r} \mathrm{u}_{0}^{r} \nabla_{y} \xi^{3_{j}}(y), \nabla_{y} \phi^{r}\right)_{Y^{s}}, \psi^{r}\right)_{\Omega} .
\end{aligned}
$$

Proof. 1 The lemma is a consequence of Lax-Milgram's thoerem.

Step 1: Problem (44): The assumptions of Lax-Milgrams are easily verified since $\kappa(y)$ is a strongly elliptic matrix, see Definition II.2.

Step 2: Problem (45): a) Continuity: For $\mathrm{v}^{r}, \mathrm{w}^{r}=\phi^{r}(y) \psi^{r}(x) \in H_{0}^{1}\left(\Omega, W_{\sharp}\left(Y^{s}\right)\right)$ we can estimate the bilinear form $\mathrm{a}_{1}^{r}$ by,

$$
\left|\mathrm{a}_{1}^{r}\left(\mathrm{v}^{r}, \mathrm{w}^{r}\right)\right|=\left|\left(\left(\nabla_{y} \mathrm{v}^{r}, \nabla_{y} \phi^{r}\right)_{Y^{s}}, \psi^{r}\right)_{\Omega}\right| \leq\left\|\mathrm{v}^{r}\right\|_{L^{2}\left(\Omega ; W_{\sharp}\left(Y^{s}\right)\right)}\left\|\phi^{r}\right\|_{W_{\sharp}\left(Y^{s}\right)}\left\|\psi^{r}\right\|_{L^{2}(\Omega)},
$$

and hence continuity follows.

b) Coercivity: For $\mathrm{v}^{r}, \mathrm{w}^{r}=\phi^{r}(y) \psi^{r}(x) \in H_{0}^{1}\left(\Omega, W_{\sharp}\left(Y^{s}\right)\right)$ we derive a lower bound by,

$$
\mathrm{a}_{1}^{r}\left(\mathrm{v}^{r}, \mathrm{v}^{r}\right)=\left(\left(\nabla_{y} \mathrm{v}^{r}, \nabla_{y} \mathrm{v}^{r}\right)_{Y^{s}}, 1\right)_{\Omega}=\left\|\nabla_{y} \mathrm{v}^{r}\right\|_{L^{2}\left(\Omega ; L^{2}\left(Y^{s}\right)\right)}^{2} .
$$

c) $\mathrm{F}_{1}^{r_{j}}$ is linear and continuous: For $\mathrm{w}^{r}=\phi^{r}(y) \psi^{r}(x) \in H_{0}^{1}\left(\Omega, W_{\sharp}\left(Y^{s}\right)\right)$ we estimate $\mathrm{F}_{1}^{r_{j}}$ by,

$$
\begin{aligned}
\left|\mathrm{F}_{1}^{r_{j}}\left(w^{r}\right)\right| & \leq\left|\left(\left(z_{r} \mathrm{u}_{0}^{r} \nabla_{y} \xi^{3_{j}}, \nabla_{y} \phi^{r}\right)_{Y^{s}}, \psi^{r}\right)_{\Omega}\right| \\
& \leq C\left\|\nabla_{y} \xi^{3_{j}}\right\|_{L^{2}\left(Y^{s}\right)}\left\|\nabla_{y} \phi^{r}\right\|_{L^{2}\left(Y^{s}\right)}\left\|\mathrm{u}_{0}^{r}\right\|_{L^{2}(\Omega)}\left\|\psi^{r}\right\|_{L^{2}(\Omega)},
\end{aligned}
$$

and continuity follows with $\xi^{3_{j}} \in W_{\sharp}(Y)$ obtained in Step 1.

(3) Problem for terms of order $\mathcal{O}(1)$ : We collect all the terms which do not contain any factor $\epsilon$. As a result we end up with the following equation

$$
\mathcal{O}(1): \quad\left\{\begin{array}{r}
-\mathcal{S}_{0}\left(\mathbf{u}_{0}, \mathbf{y}\right) \mathbf{u}_{2}=\tilde{\mathcal{S}}_{1}\left(\mathbf{u}_{0}, \mathbf{u}_{1}, \mathbf{y}\right) \mathbf{u}_{1} \\
\quad+\tilde{\mathcal{S}}_{2}\left(\mathbf{u}_{0}, \mathbf{u}_{1}, \mathbf{u}_{2}, \mathbf{y}\right) \mathbf{u}_{0}+\mathbf{I}\left(\mathbf{u}_{0}\right)+\mathbf{D}_{\mathbf{t}} \mathbf{u}_{0} \\
\mathbf{u}_{2}(\cdot, \mathbf{y}) Y \text {-periodic }
\end{array}\right.
$$


New porous medium Poisson-Nernst-Planck equations

where $\tilde{\mathcal{S}}_{1}:=\mathcal{S}_{1}\left(\mathbf{u}_{0}, \mathbf{y}\right)+\left[\mathcal{S}_{0}\left(\mathbf{u}_{1}, \mathbf{y}\right)\right]^{N}$ and $\tilde{\mathcal{S}}_{2}:=\mathcal{S}_{2}\left(\mathbf{u}_{0}, \mathbf{y}\right)+\left[\mathcal{S}_{1}\left(\mathbf{u}_{1}, \mathbf{y}\right)\right]^{N}+\left[\mathcal{S}_{0}\left(\mathbf{u}_{1}, \mathbf{y}\right)\right]^{N}$. In order to study solvability of (50) it is an advantage to write (50) explicitly for each physical quantity. For $r=1,2$ we have

$$
\mathcal{O}(1):\left\{\begin{aligned}
-\Delta_{y} \mathrm{u}_{2}^{r}= & \operatorname{div}_{y}\left(z_{r} \mathrm{u}_{0}^{r} \nabla_{y} \mathrm{u}_{2}^{3}\right) \\
& +\left\{\operatorname{div}_{x}\left(z_{r} \mathrm{u}_{0}^{r} \nabla_{y} \mathrm{u}_{1}^{3}\right)+\operatorname{div}_{y}\left(z_{r} \mathrm{u}_{0}^{r} \nabla_{x} \mathrm{u}_{1}^{3}\right)\right. \\
& \left.+\operatorname{div}_{x} \nabla_{y} \mathrm{u}_{1}^{r}+\operatorname{div}_{y} \nabla_{x} \mathrm{u}_{1}^{r}+\operatorname{div}_{y}\left(z_{r} \mathrm{u}_{1}^{r} \nabla_{y} \mathrm{u}_{1}^{3}\right)\right\} \\
& +\left\{\Delta_{x} \mathrm{u}_{0}^{r}+\operatorname{div}_{x}\left(z_{r} \mathrm{u}_{0}^{r} \nabla_{x} \mathrm{u}_{0}^{3}\right)+\operatorname{div}_{x}\left(z_{r} \mathrm{u}_{1}^{r} \nabla_{y} \mathrm{u}_{0}^{3}\right)\right. \\
& \left.+\operatorname{div}_{y}\left(z_{r} \mathrm{u}_{1}^{r} \nabla_{x} \mathrm{u}_{0}^{3}\right)+\operatorname{div}_{y}\left(z_{r} \mathrm{u}_{2}^{r} \nabla_{y} \mathrm{u}_{0}^{3}\right)\right\}-\partial_{t} \mathrm{u}_{0}^{r} \quad \text { in } \Omega^{s} \times Y^{s}, \\
-\operatorname{div}_{y}(\kappa & \left.(y) \nabla_{y} \mathrm{u}_{2}^{3}\right)=\operatorname{div}_{x}\left(\kappa(y) \nabla_{y} \mathrm{u}_{1}^{3}\right)+\operatorname{div}_{y}\left(\kappa(y) \nabla_{x} \mathrm{u}_{1}^{3}\right) \\
& +\operatorname{div}_{x}\left(\kappa(y) \nabla_{x} \mathrm{u}_{0}^{3}\right)+\left(\mathrm{u}_{0}^{1}-\mathrm{u}_{0}^{2}\right) \chi_{Y^{s}} \quad \text { in } Y .
\end{aligned}\right.
$$

Since a solvability constraint implies the effective equation for the macroscopic quantities, we first achieve the well-posedness for the system (51).

Lemma IV.3 The problem (51) $)_{2}$, i.e.,

$$
\left\{\begin{array}{l}
\text { Find } \mathrm{u}_{2}^{3}(\cdot, \cdot, y) \in W_{\sharp}(Y) \text { such that } \\
\mathrm{a}_{2}^{3}\left(\mathrm{u}_{2}^{3}, w^{3}\right)=\mathrm{F}_{2}^{3}\left(w^{3}\right)
\end{array} \forall w^{3} \in W_{\sharp}(Y),\right.
$$

has a unique solution $\mathrm{u}_{2}^{3} \in H^{1}\left(\Omega_{T}, W_{\sharp}(Y)\right)$ where we define,

$$
\begin{aligned}
\mathrm{a}_{2}^{3}\left(\mathrm{u}_{2}^{3}, w^{3}\right):= & \left(\kappa(y) \nabla_{y} \mathrm{u}_{2}^{3}, \nabla_{y} w^{3}\right)_{Y} \\
\mathrm{~F}_{2}^{3}\left(w^{3}\right):= & \left(\operatorname{div}_{x}\left(\kappa(y) \nabla_{y} \mathrm{u}_{1}^{3}\right), w^{3}\right)_{Y}-\left(\kappa(y) \nabla_{x} \mathrm{u}_{1}^{3}, \nabla_{y} w^{3}\right)_{Y} \\
& +\left(\operatorname{div}_{x}\left(\kappa(y) \nabla_{x} \mathrm{u}_{0}^{3}\right), w^{3}\right)_{Y}+\left(\mathrm{u}_{0}^{1}-\mathrm{u}_{0}^{2}, w^{3}\right)_{Y^{s}} .
\end{aligned}
$$

For $r=1,2$ equation (51) 1 , that means the following problem,

$$
\left\{\begin{array}{l}
\text { Find } \mathrm{u}_{2}^{r}(\cdot, \cdot, y) \in W_{\sharp}\left(Y^{s}\right) \text { such that } \\
\mathrm{a}_{2}^{r}\left(\mathrm{u}_{2}^{r}, w^{r}\right)=\mathrm{F}_{2}^{r}\left(w^{r}\right)
\end{array} \forall w^{r} \in W_{\sharp}\left(Y^{s}\right),\right.
$$


New porous medium Poisson-Nernst-Planck equations

has a unique solution $\mathrm{u}_{2}^{r}(\cdot, \cdot, y) \in W_{\sharp}\left(Y^{s}\right)$, where we for $z_{1}=1, z_{2}=-1$ define,

$$
\begin{aligned}
\mathrm{a}_{2}^{r}\left(\mathrm{u}_{2}^{r}, w^{r}\right):= & \left(\nabla_{y} \mathrm{u}_{2}^{r}, \nabla_{y} w^{r}\right)_{Y^{s}} \\
\mathrm{~F}_{2}^{r}\left(w^{r}\right):= & -\left(z_{r} \mathrm{u}_{0}^{r} \nabla_{y} \mathrm{u}_{2}^{3}, \nabla_{y} w^{r}\right)_{Y^{s}}+\left(\operatorname{div}_{x}\left(z_{r} \mathrm{u}_{0}^{r} \nabla_{y} \mathrm{u}_{1}^{3}\right), w^{r}\right)_{Y^{s}} \\
& -\left(z_{r} \mathrm{u}_{0}^{r} \nabla_{x} \mathrm{u}_{1}^{3}, \nabla_{y} w^{r}\right)_{Y^{s}}+\left(\operatorname{div}_{x} \nabla_{y} \mathrm{u}_{1}^{r}, w^{r}\right)_{Y^{s}}-\left(\partial_{t} \mathrm{u}_{0}^{r}, w^{r}\right)_{Y^{s}} \\
& -\left(\nabla_{x} \mathrm{u}_{1}^{r}, \nabla_{y} w^{r}\right)_{Y^{s}}-\left(z_{r} \mathrm{u}_{1}^{r} \nabla_{y} \mathrm{u}_{1}^{3}, \nabla_{y} w^{r}\right)_{Y^{s}}+\left(\Delta_{x} \mathrm{u}_{0}^{r}, w^{r}\right)_{Y^{s}} \\
& +\left(\operatorname{div}_{x}\left(z_{r} \mathrm{u}_{0}^{r} \nabla_{x} \mathrm{u}_{0}^{r}\right), w^{r}\right)_{Y^{s}}-\left(z_{r} \mathrm{u}_{1}^{r} \nabla_{x} \mathrm{u}_{0}^{3}, \nabla_{y} w^{r}\right)_{Y^{s}} .
\end{aligned}
$$

Proof. 2 Step 1: Theorem II.3 and LemmaII.1 immediately provide existence and uniqueness of a solution $\mathrm{u}_{2}^{3} \in W_{\sharp}(Y)$, if we have,

$$
\left\langle\mathrm{F}_{2}^{3}, 1\right\rangle_{\left(H_{\sharp}^{1}\left(Y^{s}\right)\right)^{\prime}, H_{\sharp}^{1}\left(Y^{s}\right)}=0 .
$$

Equation (56) reads in terms of physical quantities as,

$$
\left(\operatorname{div}_{x}\left(\kappa(y) \nabla_{y} \mathrm{u}_{1}^{3}\right), 1\right)_{Y}-\left(\operatorname{div}_{x}\left(\kappa(y) \nabla_{x} \mathrm{u}_{0}^{3}\right), 1\right)_{Y}+\left(\mathrm{u}_{0}^{1}-\mathrm{u}_{0}^{2}\right)_{Y^{s}}=0 .
$$

This equation defines the upscaled formulation (25) $)_{2}$ for the electric potential such that (56) holds true.

Step 2: Again, solvability follows by Theorem II.3 after verification of $\mathrm{F}_{2}^{r} \in\left(W_{\sharp}\left(Y^{s}\right)\right)^{\prime}$. Due to Theorem II.3 and Lemma II.1, it must hold for $r=1,2$ that

$$
\left\langle\mathrm{F}_{2}^{r}, 1\right\rangle_{\left(H_{\sharp}^{1}\left(Y^{s}\right)\right)^{\prime}, H_{\sharp}^{1}\left(Y^{s}\right)}=0,
$$

which reads in explicit form as follows,

$$
\begin{aligned}
\left(\operatorname{div}_{x} \nabla_{y} \mathrm{u}_{1}^{r}, 1\right)_{Y^{s}} & +\left(\Delta_{x} \mathrm{u}_{0}^{r}, 1\right)_{Y^{s}}-\left(\partial_{t} \mathrm{u}_{0}^{r}, 1\right)_{Y^{s}} \\
& +\left(\operatorname{div}_{x}\left(z_{r} \mathrm{u}_{0}^{r} \nabla_{y} \mathrm{u}_{1}^{3}\right), 1\right)_{Y^{s}}+\left(\operatorname{div}_{x}\left(z_{r} \mathrm{u}_{0}^{r} \nabla_{y} \mathrm{u}_{0}^{3}\right), 1\right)_{Y^{s}}=0 .
\end{aligned}
$$

Since we use (59) represents the by Lemma III.4 well-posed effective model, we herewith guarantee that (58) holds.

With representation (42) 2 we can rewrite (57) in the following way,

$$
-\sum_{i, j, k=1}^{N}\left(\partial_{x_{i}}\left(\kappa(y)\left(\delta_{i k}-\delta_{i j} \partial_{y_{j}} \xi^{3_{k}}\right) \partial_{x_{k}} \mathrm{u}_{0}^{3}\right), 1\right)_{Y}=\left|Y^{s}\right|\left(\mathrm{u}_{0}^{1}-\mathrm{u}_{0}^{2}\right)
$$


New porous medium Poisson-Nernst-Planck equations

Using (38) allows us to write (60) more intuitively by,

$$
|Y| \sum_{i, j, k=1}^{N} \epsilon_{i k}^{0} \frac{\partial^{2} \mathrm{u}_{0}^{3}}{\partial x_{i} \partial x_{k}}=\left|Y^{s}\right|\left(\mathrm{u}_{0}^{1}-\mathrm{u}_{0}^{2}\right)
$$

such that $\boldsymbol{\epsilon}^{0}:=\left\{\epsilon_{i k}^{0}\right\}_{1 \leq i, k \leq N}$ is defined by

$$
\epsilon_{i k}^{0}:=-\frac{1}{|Y|}\left(\left(\kappa(y)\left(\delta_{i k}-\delta_{i j} \partial_{y_{j}} \xi^{3_{k}}\right)\right), 1\right)_{Y} .
$$

We can write down in the same way effective equations for equations (154) by using (42) 1 . That means we rewrite (59) in the following way,

$$
\begin{aligned}
& \left(\partial_{x_{i}}\left(\delta_{i k} \partial_{x_{k}} \mathrm{u}_{0}^{r}\right), 1\right)_{Y^{s}}+\left(\partial_{x_{i}}\left(\left\{-\delta_{i j} \partial_{y_{j}} \xi^{r_{k}}\right\} \partial_{x_{k}} \mathrm{u}_{0}^{3}\right), 1\right)_{Y^{s}}-\left(\partial_{t} \mathrm{u}_{0}^{r}, 1\right)_{Y^{s}} \\
& +\left(\partial_{x_{i}}\left(z_{r} \mathrm{u}_{0}^{r}\left\{\delta_{i k}-\delta_{i j} \partial_{y_{j}} \xi^{3_{k}}\right\} \partial_{x_{k}} \mathrm{u}_{0}^{3}\right), 1\right)_{Y^{s}}=0 .
\end{aligned}
$$

If we apply (39) we can even further simplify (63) to

$$
\begin{aligned}
\left|Y^{s}\right| \partial_{t} \mathrm{u}_{0}^{r}-\left|Y^{s}\right| \Delta \mathrm{u}_{0}^{r} & +\partial_{x_{i}}\left(\int_{Y^{s}}\left\{\delta_{i j} \partial_{y_{j}} \xi^{r_{k}}(x, y)\right\} d y \partial_{x_{k}} \mathrm{u}_{0}^{3}\right) \\
& -\partial_{x_{i}}\left(z_{r} \mathrm{u}_{0}^{r} \int_{Y^{s}}\left\{\delta_{i k}-\delta_{i j} \partial_{y_{j}} \xi^{3{ }_{k}}(y)\right\} d y \partial_{x_{k}} \mathrm{u}_{0}^{3}\right)=0 .
\end{aligned}
$$

As a consequence of (64) we obtain the effective diffusion-related tensor $\mathbb{D}^{r}:=\left\{\mathrm{D}_{i k}^{r}\right\}_{1 \leq i, k \leq N}$, i.e.,

$$
\mathrm{D}_{i k}^{r}:=\frac{1}{|Y|} \int_{Y^{s}}\left\{\delta_{i j} \partial_{y_{j}} \xi^{r_{k}}(t, x, y)\right\} d y \quad \forall i, k=1, \ldots, N
$$

and the electro-diffusion-related tensor $\mathbb{M}:=\left\{\mathrm{M}_{i k}\right\}_{1 \leq i, k \leq N}$, i.e.,

$$
\mathrm{M}_{i k}:=\frac{1}{|Y|} \int_{Y^{s}}\left\{\delta_{i k}-\delta_{i j} \partial_{y_{j}} \xi^{3_{k}}(y)\right\} d y \quad \forall i, k=1, \ldots, N
$$

The definitions (65) and (66) finally provide the porous media approximation of the NernstPlanck-Poisson equations,

upscaled model : $\left\{\begin{array}{l}p \partial_{t} \mathrm{u}_{0}^{r}-p \Delta \mathrm{u}_{0}^{r}+\operatorname{div}\left(\mathbb{D}^{r}(t, x) \nabla \mathrm{u}_{0}^{3}\right)-\operatorname{div}\left(z_{r} \mathrm{u}_{0}^{r} \mathbb{M} \nabla \mathrm{u}_{0}^{3}\right)=0 \\ -\operatorname{div}\left(\epsilon^{0} \nabla \mathrm{u}_{0}^{3}\right)=p\left(\mathrm{u}_{0}^{1}-\mathrm{u}_{0}^{2}\right)\end{array}\right.$

where $p:=\frac{\left|Y^{s}\right|}{|Y|}$ is the porosity, $r=1,2, z_{1}=1$, and $z_{2}=-1$. 
New porous medium Poisson-Nernst-Planck equations

(4) Derivation of the second order correctors: In order to compute the second order corrector for $\mathrm{u}_{0}^{3}$ we use $(\underline{42})_{2}$ in equation $(\underline{51})_{2}$, i.e.,

$$
\begin{aligned}
-\operatorname{div}_{y}\left(\kappa(y) \nabla_{y} \mathrm{u}_{2}^{3}\right) & =-\sum_{i, j, k=1}^{N} \partial_{x_{i}}\left(\kappa(y) \delta_{i j} \partial_{y_{j}} \xi^{3_{k}} \partial_{x_{k}} \mathrm{u}_{0}^{3}\right) \\
& -\sum_{i, j, k=1}^{N} \partial_{y_{i}}\left(\kappa(y) \delta_{i j} \partial_{x_{j}}\left(\xi^{3 k_{k}} \partial_{x_{k}} \mathrm{u}_{0}^{3}\right)\right. \\
& +\operatorname{div}_{x}\left(\kappa(y) \nabla_{x} \mathrm{u}_{0}^{3}\right)+\left(\mathrm{u}_{0}^{1}-\mathrm{u}_{0}^{2}\right) \chi_{Y^{s}} \quad \text { in } Y .
\end{aligned}
$$

Inserting equation (61) into (68) leads to the following problem,

$$
\begin{aligned}
-\operatorname{div}_{y}\left(\kappa(y) \nabla_{y} \mathrm{u}_{2}^{3}\right) & =-\sum_{k, l=1}^{N} \epsilon_{k l}^{0} \frac{\partial^{2} \mathrm{u}_{0}^{3}}{\partial x_{k} \partial x_{l}}-\sum_{i, j, k=1}^{N} \kappa(y) \delta_{k j} \partial_{y_{j}} \xi^{3} \frac{\partial^{2} \mathrm{u}_{0}^{3}}{\partial x_{l} \partial x_{k}} \\
& -\sum_{i, j, k=1}^{N} \partial_{y_{i}}\left(\kappa(y) \delta_{i j} \xi^{3 k}\right) \frac{\partial^{2} \mathrm{u}_{0}^{3}}{\partial x_{l} \partial x_{k}}+\sum_{j, l=1}^{N} \kappa(y) \delta_{j l} \frac{\partial^{2} \mathrm{u}_{0}^{3}}{\partial x_{j} \partial x_{l}} \quad \text { in } Y .
\end{aligned}
$$

With equation (69) the right-hand side $\mathrm{F}_{2}^{3}$ in $(53)_{2}$ can be rewritten by,

$$
\begin{aligned}
\left\langle\mathrm{F}_{2}^{3}, w^{3}\right\rangle_{\left(W_{\sharp}(Y)\right)^{\prime}, W_{\sharp}(Y)} & =\sum_{k, l=1}^{N}\left[-\epsilon_{k l}^{0} \int_{Y} w^{3} d y-\sum_{i, j=1}^{N} \int_{Y} \partial_{y_{i}}\left(\kappa(y) \delta_{i j} \delta_{k j} \xi^{3}\right) w^{3} d y\right. \\
& \left.-\sum_{j, k, l=1}^{N} \int_{Y} \kappa(y) \delta_{k j} \partial_{y_{j}}\left(\xi^{3_{l}}-y_{l}\right) w^{3} d y\right] \frac{\partial^{2} \mathrm{u}_{0}^{3}}{\partial x_{k} \partial x_{l}} \quad \forall w^{3} \in W_{\sharp}(Y) .
\end{aligned}
$$

The same arguments as those for (42) suggest to look for a function $\mathrm{u}_{2}^{3}$ of the following form,

$$
\mathrm{u}_{2}^{3}(t, x, y):=\sum_{k, l=1}^{N} \zeta^{3 k l}(y) \frac{\partial^{2} \mathrm{u}_{0}^{3}}{\partial x_{k} \partial x_{l}}
$$

where $\zeta^{3 k l}$ is the solution of,

$$
-\operatorname{div}_{y}\left(\kappa(y) \nabla_{y} \zeta^{3_{k l}}\right)=-\epsilon_{k l}^{0}-\sum_{i, j=1}^{N} \partial_{y_{i}}\left(\kappa(y) \delta_{i j} \delta_{k j} \xi^{3_{l}}\right)-\sum_{j=1}^{N} \kappa(y) \delta_{k j} \partial_{y_{j}}\left(\xi^{3_{l}}-y_{l}\right) \quad \text { in } Y .
$$

Lemma IV.4 There exists a unique $\zeta^{3_{k l}}$ for $1 \leq k, l \leq N$ that solves equation (72) in the weak sense, that means, $\zeta^{3 k l}$ is a unique solution of the following problem,

$$
\left\{\begin{array}{l}
\text { Find } \zeta^{3_{k l}} \in W_{\sharp}(Y) \text { such that } \\
\mathrm{a}_{2}^{3}\left(\zeta^{3 k l}, w^{3}\right)=\mathrm{F}_{2}^{3_{k l}}\left(w^{3}\right) \quad \forall w^{3} \in W_{\sharp}(Y) .
\end{array}\right.
$$


New porous medium Poisson-Nernst-Planck equations

where we define,

$$
\begin{aligned}
\mathrm{a}_{2}^{3}\left(\zeta^{3_{k l}}, w^{3}\right):= & \left(\kappa(y) \nabla_{y} \zeta^{3_{k l}}, \nabla_{y} w^{3}\right)_{Y} \\
\mathrm{~F}_{2}^{3_{k l}}\left(w^{3}\right):= & -\left(\epsilon_{k l}^{0}, w^{3}\right)_{Y}-\sum_{i, j=1}^{N}\left(\partial_{y_{i}}\left(\kappa(y) \delta_{i j} \delta_{k j} \xi^{3 l}\right), w^{3}\right)_{Y} \\
& -\sum_{j=1}^{N}\left(\kappa(y) \delta_{k j} \partial_{y_{j}}\left(\xi^{3_{l}}-y_{l}\right), w^{3}\right)_{Y}
\end{aligned}
$$

Proof. 3 The existence and uniqueness is an immediate consequence of Theorem (II.3) and Lemma (II.1).

The same considerations as those for the derivation of (172) can be applied to (55) (or to (51) in the context of its classical formulation). With (771) and (42) we can rewrite (51) as,

$$
\begin{aligned}
-\Delta_{y} \mathrm{u}_{2}^{r} & =\frac{1}{p} \sum_{k, l=1}^{N} \mathrm{D}_{k l}^{r} \frac{\partial^{2} \mathrm{u}_{0}^{3}}{\partial x_{k} \partial x_{l}}-\frac{1}{p} \sum_{k, l=1}^{N} \partial_{x_{k}}\left(z_{r} \mathrm{u}_{0}^{r} \mathrm{M}_{k l} \partial_{x_{l}} \mathrm{u}_{0}^{3}\right)-\sum_{k, l=1}^{N} \frac{\partial^{2} \mathrm{u}_{0}^{r}}{\partial x_{r} \partial x_{l}} \\
& -\sum_{j, k, l=1}^{N} \delta_{k j} \partial_{y_{j}} \xi^{r_{l}} \frac{\partial^{2} \mathrm{u}_{0}^{3}}{\partial x_{l} \partial x_{k}}-\sum_{i, j, k=1}^{N} \partial_{y_{i}}\left(\delta_{i j} \xi^{r_{k}}\right) \frac{\partial^{2} \mathrm{u}_{0}^{3}}{\partial x_{j} \partial x_{k}}+\sum_{j, l=1}^{N} \delta_{j l} \frac{\partial^{2} \mathrm{u}_{0}^{r}}{\partial x_{j} \partial x_{l}} \\
& -\sum_{j, k, l=1}^{N}\left\{z_{r} \delta_{k j} \partial_{y_{j}} \xi^{3 l} \frac{\partial \mathrm{u}_{0}^{r}}{\partial x_{l}} \frac{\partial \mathrm{u}_{0}^{3}}{\partial x_{k}}+z_{r} \mathrm{u}_{0}^{r} \delta_{k j} \partial_{y_{j}} \xi^{3} \frac{\partial^{2} \mathrm{u}_{0}^{3}}{\partial x_{l} \partial x_{k}}\right\} \\
& +\sum_{i, j, k, l=1}^{N} \partial_{y_{i}}\left(\delta_{i j} \partial_{y_{j}} \zeta^{3 k_{l}}\right) z_{r} \mathrm{u}_{0}^{r} \frac{\partial^{2} \mathrm{u}_{0}^{3}}{\partial x_{k} \partial x_{l}}-\sum_{i, j, k=1}^{N} \partial_{y_{i}}\left(\delta_{i j} \xi^{r_{k}}\right) z_{r} \frac{\partial \mathrm{u}_{0}^{r}}{\partial x_{k}} \frac{\partial \mathrm{u}_{0}^{3}}{\partial x_{j}} \\
& -\sum_{i, j, k=1}^{N} z_{r} \mathrm{u}_{0}^{r} \partial_{y_{i}}\left(\delta_{i j} \xi^{3{ }_{k}}\right) \frac{\partial^{2} \mathrm{u}_{0}^{3}}{\partial x_{k} \partial x_{j}}+\sum_{i, j, k, l=1}^{N} z_{r} \partial_{y_{i}}\left(\delta_{i j} \xi^{r_{k}} \partial_{y_{j}} \xi^{3 l}\right) \frac{\partial \mathrm{u}_{0}^{r}}{\partial x_{k}} \frac{\partial \mathrm{u}_{0}^{3}}{\partial x_{l}} \\
& +\sum_{j, l=1}^{N}\left\{z_{r} \delta_{j l} \frac{\partial \mathrm{u}_{0}^{r}}{\partial x_{j}} \frac{\partial \mathrm{u}_{0}^{3}}{\partial x_{l}}+z_{r} \mathrm{u}_{0}^{r} \delta_{j l} \frac{\partial^{2} \mathrm{u}_{0}^{3}}{\partial x_{j} \partial x_{l}}\right\} \text { in } Y^{s} .
\end{aligned}
$$

We make now a corresponding Ansatz for the functions $\mathrm{u}_{2}^{r}$ with the indices $r=1,2$ under the same considerations as those for (71), i.e.,

$$
\mathrm{u}_{2}^{r}(t, x, y)=\sum_{k, l=1}^{N} \zeta^{r k l}(t, x, y) \mathrm{u}_{0}^{r}
$$

After inserting definition (76) into (75) we obtain an equation for the second order corrector 
New porous medium Poisson-Nernst-Planck equations

functions $\zeta^{r k l}$, that means,

$$
\begin{aligned}
-\sum_{k, l=1}^{N} \Delta_{y} \zeta^{r_{k l}} \mathrm{u}_{0}^{r}=\sum_{k, l=1}^{N}\left[\frac{1}{p} \mathrm{D}_{k l}^{r}-\sum_{j=1}^{N} \delta_{k j} \partial_{y_{j}}\left(\xi^{r_{l}}\right)-\sum_{i, j=1}^{N} \partial_{y_{i}}\left(\delta_{i j} \delta_{k j} \xi^{r_{k}}\right)\right] \frac{\partial^{2} \mathrm{u}_{0}^{3}}{\partial x_{k} \partial x_{l}} \\
+\sum_{k, l=1}^{N}\left[-\frac{z_{r}}{p} \mathrm{M}_{k l}-\sum_{j=1}^{N} z_{r} \delta_{k j} \partial_{y_{j}}\left(\xi^{3_{l}}-y_{l}\right)\right. \\
\left.+\sum_{i, j=1}^{N} z_{r} \partial_{y_{i}}\left(\delta_{i j} \delta_{k j} \xi^{r_{k}}\left(\partial_{y_{j}} \xi^{3_{l}}-1\right)\right)\right] \frac{\partial \mathrm{u}_{0}^{r}}{\partial x_{k}} \frac{\partial \mathrm{u}_{0}^{3}}{\partial x_{l}} \\
\quad+\sum_{k, l=1}^{N}\left[-\sum_{j=1}^{N} \delta_{k j} \partial_{y_{j}}\left(\xi^{3_{l}}-y_{l}\right)\right. \\
\left.\quad-\sum_{i, j=1}^{N} \partial_{y_{i}}\left(\delta_{i j} \delta_{k j}\left(\xi^{3 k_{k}}-\partial_{y_{j}} \zeta^{3_{k l}}\right)\right)\right] z_{r} \mathrm{u}_{0}^{r} \frac{\partial^{2} \mathrm{u}_{0}^{3}}{\partial x_{k} \partial x_{l}}+\sum_{k, l, j=1}^{N}\left(\frac{\partial y_{l}}{\partial y_{j}}-\delta_{k l}\right) \frac{\partial^{2} \mathrm{u}_{0}^{r}}{\partial x_{k} \partial x_{l}} .
\end{aligned}
$$

In order to guarantee solvability of equation (77), we assume that $\mathrm{u}_{0}^{r}>0$ for $\mathrm{u}_{0}^{r}(0, x) \geq \eta>0$. This can be obtained by special test function techniques as applied in $\underline{41}$ to prove nonnegativity of solutions. Existence and uniqueness of the corrector functions defined by equations (72) and (77) is achieved in the following

Lemma IV.5 Let $\mathrm{u}_{0}^{r} \in V\left(\Omega_{T}\right), \mathrm{u}_{0}^{r}(t, x)>0$ for all $(t, x) \in \Omega_{T}$ and $1 \leq r \leq N$, and assume that the reference cells $Y$ are in local thermodynamic equilibrium, see Definition (III.1). Then, there exists a unique $\zeta^{r_{k l}}$ for $1 \leq k, l \leq N$ that solves equation (72) in the weak sense. That means, $\zeta^{r}$ is a unique solution of the following problem,

$$
\left\{\begin{array}{lr}
\text { Find } \zeta^{r k l}(t, x, y) \in V\left(\Omega_{T}, W_{\sharp}\left(Y^{s}\right)\right) & \text { for } 1 \leq k, l \leq N \text { such that } \\
a_{2}^{r}\left(\zeta^{r_{k l}}, w^{r}\right)=\mathrm{F}_{2}^{r_{k l}}\left(w^{r}\right) & \forall w^{r} \in H_{0}^{1}\left(\Omega, W_{\sharp}\left(Y^{s}\right)\right) .
\end{array}\right.
$$


New porous medium Poisson-Nernst-Planck equations

where we define for $w^{r}:=\phi^{r}(x) \psi^{r}(y)$,

$$
\begin{aligned}
\mathrm{a}_{2}^{r}\left(\zeta^{r_{k l}}, w^{r}\right): & \left(\left(\mathrm{u}_{0}^{r} \kappa(y) \nabla_{y} \zeta^{r_{k l}}, \nabla_{y} \phi^{r}\right)_{Y^{s}}, \psi^{r}\right)_{\Omega} \\
\mathrm{F}_{2}^{r_{k l}}\left(w^{r}\right): & =\left(\left(\mathrm{D}_{k l}^{r}-\sum_{j=1}^{N} \delta_{k j} \partial_{y_{j}}\left(\xi^{r_{l}}-y_{l}\right)-\sum_{i, j=1}^{N} \partial_{y_{i}}\left(\delta_{i j} \delta_{k j} \xi^{r_{k}}\right), \phi^{r}\right)_{Y^{s}} \frac{\partial^{2} \mathrm{u}_{0}^{3}}{\partial x_{k} \partial x_{l}}\right. \\
& +\left(-\mathrm{M}_{k l}-\sum_{j=1}^{N} \delta_{k j} \partial_{y_{j}}\left(\xi^{3 l}-y_{l}\right)\right. \\
& \left.+\sum_{i, j=1}^{N} \partial_{y_{i}}\left(\delta_{i j} \delta_{k j} \xi^{r_{k}}\left(\partial_{y_{j}} \xi^{3_{l}}-1\right)\right), \phi^{r}\right)_{Y^{s}} z_{r}\left(\frac{\partial \mathrm{u}_{0}^{r}}{\partial x_{k}} \frac{\partial \mathrm{u}_{0}^{3}}{\partial x_{l}}\right) \\
& +\sum_{j=1}^{N}\left(\frac{\partial y_{l}}{\partial y_{j}}-\delta_{k l}\right)_{Y^{s}} \frac{\partial^{2} \mathrm{u}_{0}^{r}}{\partial x_{k} \partial x_{l}} \\
& +\left(-\mathrm{M}_{k l}-\sum_{j=1}^{N} \delta_{k j} \partial_{y_{j}}\left(\xi^{3 l}-y_{l}\right)\right. \\
& \left.\left.-\sum_{i, j=1}^{N} \partial_{y_{i}}\left(\delta_{i j} \delta_{k j}\left(\xi^{33_{k}}-\partial_{y_{j}} \zeta^{3 k_{k l}}\right)\right), \phi^{r}\right)_{Y^{s}} z_{r} \mathrm{u}_{0}^{r} \frac{\partial^{2} \mathrm{u}_{0}^{3}}{\partial x_{k} \partial x_{l}}, \psi^{r}\right)_{\Omega}
\end{aligned}
$$

Proof. 4 We apply Lax-Milgram's theorem in three steps:

a) Continuity: For $v(x, y), w(x, y) \in H_{0}^{1}\left(\Omega ; W_{\sharp}\left(Y^{s}\right)\right)$ we have

$$
\left|\mathrm{a}_{2}^{r}(v, w)\right|=\left|\left(\kappa(y) \nabla_{y} v, \nabla_{y} w\right)_{Y^{s}, \Omega}\right| \leq C\left\|\nabla_{y} v\right\|_{H^{1}\left(\Omega ; L^{2}\left(Y^{s}\right)\right)}\left\|\nabla_{y} w\right\|_{H^{1}\left(\Omega ; L^{2}\left(Y^{s}\right)\right)},
$$

which proves continuity since $\left\|\nabla_{y} v\right\|_{L^{2}\left(Y^{s}\right)}$ defines a norm on $W_{\sharp}(Y)$ and $\kappa \in M(c, C, Y)$.

b) Coercivity: For $v(x, y) \in H_{0}^{1}\left(\Omega ; W_{\sharp}\left(Y^{s}\right)\right)$ we can estimate

$$
\left|\mathrm{a}_{2}^{r}(v, v)\right|=\left|\left(\mathrm{u}_{0}^{r} \kappa(y) \nabla_{y} v, \nabla_{y} v\right)_{Y^{s}, \Omega}\right| \geq c\left\|\nabla_{y} v\right\|_{H^{1}\left(\Omega ; L^{2}\left(Y^{s}\right)\right)}^{2} .
$$

c) $\mathrm{F}_{2}^{r_{k l}}$ is linear and continuous: In order to keep the clear representation we only study the two critical terms, i.e.,

$$
\begin{aligned}
(I) & :=\left(\left(\delta_{i j} \delta_{k j} \xi^{r_{k}}\left(\partial_{y_{j}} \xi^{3_{l}}-1\right), \partial_{y_{i}} \phi^{r}\right)_{Y^{s}}\left(\frac{\partial \mathrm{u}_{0}^{r}}{\partial x_{k}} \frac{\partial \mathrm{u}_{0}^{3}}{\partial x_{l}}\right), \psi^{r}\right)_{\Omega} \\
(I I) & :=\left(\left(\delta_{i j} \delta_{k j}\left(\xi^{3_{k}}-\partial_{y_{j}} \zeta^{3 k l}\right), \partial_{y_{i}} \phi^{r}\right)_{Y^{s}} z_{r} \mathrm{u}_{0}^{r} \frac{\partial^{2} \mathrm{u}_{0}^{3}}{\partial x_{k} \partial x_{l}}, \psi^{r}\right)_{\Omega}
\end{aligned}
$$

We need to show that $|(I)|,|(I I)| \leq C\|w\|_{H^{1}\left(\Omega_{T} ; W_{\sharp}\left(Y^{s}\right)\right)}$. The second term $(I I)$ can be esti- 
New porous medium Poisson-Nernst-Planck equations

mated for $w^{r}(x, y)=\phi^{r}(x) \psi^{r}(y) \in H_{0}^{1}\left(\Omega ; W_{\sharp}\left(Y^{s}\right)\right)$ by,

$$
\begin{gathered}
|(I I)| \leq C\left(\left\|\xi^{3 k}\right\|_{H^{1}\left(\Omega ; L^{2}\left(Y^{s}\right)\right)}+\left\|\nabla_{y} \zeta^{3_{k l}}\right\|_{L^{2}\left(Y^{s}\right)}\right)\left\|\nabla_{y} \phi^{r}\right\|_{L^{2}\left(Y^{s}\right)} \\
\left\|\frac{\partial^{2} \mathrm{u}_{0}^{3}}{\partial x_{k} \partial x_{l}}\right\|_{L^{2}(\Omega)}\left\|\mathrm{u}_{0}^{r}\right\|_{H^{1}(\Omega)}\left\|\psi^{r}\right\|_{H^{1}(\Omega)} .
\end{gathered}
$$

With the regularity available for $\xi, \zeta$ and $\mathrm{u}_{0}^{r}$, the continuity of the second term (II) follows. We estimate the first term (I) by,

$$
\begin{aligned}
|(I)| \leq & C\left\|\xi^{r_{k}}\right\|_{H^{1}\left(\Omega ; W_{\sharp}\left(Y^{s}\right)\right)}\left(\left\|\nabla_{y} \xi^{3_{l}}\right\|_{L^{3}\left(Y^{s}\right)}\right. \\
& +1)\left\|\nabla_{y} \phi^{r}\right\|_{L^{2}\left(Y^{s}\right)}\left\|\mathrm{u}_{0}^{3}\right\|_{H^{2}(\Omega)}\left\|\mathrm{u}_{0}^{r}\right\|_{H^{1}(\Omega)}\left\|\psi^{r}\right\|_{H^{1}(\Omega)},
\end{aligned}
$$

which implies the desired continuity.

\section{PROOF OF LEMMA III.4; WELL-POSEDNESS OF THE UPSCALED MODEL}

For each $\mathrm{v}^{r} \in V\left(\Omega_{T}\right), r=1,2$, consider the linear system

$$
\begin{cases}p \partial_{t} \mathrm{u}^{r}-p \Delta \mathrm{u}^{r}=-\operatorname{div}\left(\overline{\mathbb{D}}^{r}(t, x) \nabla \mathrm{v}^{3}\right)+\operatorname{div}\left(z_{r} \mathrm{v}^{r} \mathbb{M} \nabla \mathrm{v}^{3}\right) & \text { in } \Omega_{T}, \\ -\operatorname{div}\left(\epsilon^{0} \nabla \mathrm{v}^{3}\right)=p\left(\mathrm{v}^{1}-\mathrm{v}^{2}\right) & \text { in } \Omega_{T},\end{cases}
$$

where $\overline{\mathbb{D}}^{r}(t, x)$ indicates that its $t$ and $x$ dependence originate from $\mathrm{v}^{r}$ for $r=1,2$. The choice of $\mathrm{v}^{r} \in V\left(\Omega_{T}\right)$ guarantees that the right-hand side in $(85)_{1}$ is in $L^{2}(\Omega)$. Hence, there exists a unique solution $\mathrm{u}^{r} \in L^{2}\left(0, T ; H_{0}^{2}(\Omega)\right), \partial_{t} \mathrm{u}^{r} \in L^{2}\left(0, T ; L^{2}(\Omega)\right)$ by standard parabolic theory.

In the same way, take $\tilde{\mathrm{v}}^{r} \in V\left(\Omega_{T}\right), r=1,2$, and let $\tilde{\mathrm{u}}^{r}$ solve,

$$
\begin{cases}p \partial_{t} \tilde{\mathrm{u}}^{r}-p \Delta \tilde{\mathrm{u}}^{r}=-\operatorname{div}\left(\tilde{\mathbb{D}}^{r}(t, x) \nabla \tilde{\mathrm{v}}^{3}\right)+\operatorname{div}\left(z_{r} \tilde{\mathrm{v}}^{r} \mathbb{M} \nabla \tilde{\mathrm{v}}^{3}\right) & \text { in } \Omega_{T}, \\ -\operatorname{div}\left(\boldsymbol{\epsilon}^{0} \nabla \tilde{\mathrm{v}}^{3}\right)=p\left(\tilde{\mathrm{v}}^{1}-\tilde{\mathrm{v}}^{2}\right) & \text { in } \Omega_{T} .\end{cases}
$$

After subtracting (86) from (85), we obtain the following equation for $\hat{\mathrm{u}}^{r}:=\mathrm{u}^{r}-\tilde{\mathrm{u}}^{r}$, $\hat{\mathbb{D}}^{0, r}:=\overline{\mathbb{D}}^{0, r}-\tilde{\mathbb{D}}^{0, r}$, for $r=1,2$, i.e.,

$$
\left\{\begin{array}{rlrl}
p \partial_{t} \hat{\mathrm{u}}^{r}-p \Delta \hat{\mathrm{u}}^{r}= & -\operatorname{div}\left(\hat{\mathbb{D}}^{0, r}(t, x) \nabla \overline{\mathrm{v}}^{3}\right)-\operatorname{div}\left(\tilde{\mathbb{D}}^{0, r}(t, x) \nabla \hat{\mathrm{v}}^{3}\right) & \\
& +z_{r} \operatorname{div}\left(\hat{\mathrm{v}}^{r} \mathbb{M} \nabla \overline{\mathrm{v}}^{3}+\tilde{\mathrm{v}}^{r} \mathbb{M} \nabla \hat{\mathrm{v}}^{3}\right) & & \text { in } \Omega_{T}, \\
-\operatorname{div}\left(\boldsymbol{\epsilon}^{0} \nabla \hat{\mathrm{v}}^{3}\right)= & p\left(\hat{\mathrm{v}}^{1}-\hat{\mathrm{v}}^{2}\right) & & \text { in } \Omega_{T} .
\end{array}\right.
$$


The test function $\hat{\mathrm{u}}^{r}, r=1,2$, in (87) together with Sobolev inqualities and $L^{p}$-interpolation estimates induce the following inequality,

$$
\begin{aligned}
\frac{p}{2} \frac{d}{d t}\left(\left\|\hat{\mathrm{u}}^{1}\right\|^{2}+\left\|\hat{\mathrm{u}}^{2}\right\|^{2}\right) & +\left(p-\frac{\alpha}{2}\right)\left(\left\|\nabla \hat{\mathrm{u}}^{1}\right\|^{2}+\left\|\nabla \hat{\mathrm{u}}^{2}\right\|^{2}\right) \\
& \leq C\left(\alpha, \epsilon, \hat{\mathrm{v}}^{r}, \tilde{\mathrm{v}}^{r}\right)\left(\left\|\hat{\mathrm{v}}^{1}\right\|^{2}+\left\|\hat{\mathrm{v}}^{2}\right\|^{2}\right)+\epsilon\left(\left\|\nabla \hat{\mathrm{v}}^{1}\right\|^{2}+\left\|\nabla \hat{\mathrm{v}}^{2}\right\|^{2}\right),
\end{aligned}
$$

which is a consequence of the following estimates,

$$
\begin{aligned}
\left|\left(\hat{\mathbb{D}}^{0, r} \nabla \overline{\mathrm{v}}^{3}, \nabla \hat{\mathrm{u}}^{r}\right)\right| & \leq C\left\|\overline{\mathrm{v}}^{3}\right\|_{H^{2}} \max _{1 \leq k, l \leq N}\left\|\hat{\mathrm{D}}_{k l}^{0, r}\right\|_{L^{3}}\left\|\nabla \hat{\mathrm{u}}^{r}\right\| \\
& \leq C\left\|\overline{\mathrm{v}}^{3}\right\|_{H^{2}}\left\|\hat{\mathrm{v}}^{r}\right\|^{1 / 2}\left\|\nabla \hat{\mathrm{v}}^{r}\right\|^{1 / 2}\left\|\nabla \hat{\mathrm{u}}^{r}\right\| \\
& \leq C(\alpha, \epsilon)\left\|\overline{\mathrm{v}}^{3}\right\|_{H^{2}}^{2}\left\|\hat{\mathrm{v}}^{r}\right\|^{2}+\epsilon\left\|\nabla \hat{\mathrm{v}}^{r}\right\|^{2}+\frac{\alpha}{8}\left\|\nabla \hat{\mathrm{u}}^{r}\right\|^{2},
\end{aligned}
$$

and

$$
\begin{aligned}
\left|\left(\tilde{\mathbb{D}}^{0, r} \nabla \hat{\mathrm{v}}^{3}, \nabla \hat{\mathrm{u}}^{r}\right)\right| & \leq C(\alpha)\left\|\tilde{\mathrm{v}}^{r}\right\|_{H^{1}}^{2}\left(\left\|\Delta \hat{\mathrm{v}}^{3}\right\|\left\|\nabla \hat{\mathrm{v}}^{3}\right\|\right)+\frac{\alpha}{8}\|\nabla \hat{\mathrm{u}}\|^{2} \\
& \leq C(\alpha)\left\|\tilde{\mathrm{v}}^{r}\right\|_{H^{1}}^{2}\left\|\nabla \hat{\mathrm{v}}^{3}\right\|\left(\left\|\hat{\mathrm{u}}^{1}\right\|^{2}+\left\|\hat{\mathrm{v}}^{2}\right\|^{2}\right)+\frac{\alpha}{8}\|\nabla \hat{\mathrm{u}}\|^{2} .
\end{aligned}
$$

It is immediately clear that the terms containing $\mathbb{M}$ can be controlled by the same bounds, since $\mathbb{M}$ is just a constant matrix. After integrating (88) with respect to time we get,

$$
\begin{aligned}
& \frac{p}{2}\left(\left\|\hat{\mathrm{u}}^{1}\right\|_{L^{\infty}\left(0, T ; L^{2}(\Omega)\right)}^{2}+\left\|\hat{\mathrm{u}}^{2}\right\|_{L^{\infty}\left(0, T ; L^{2}(\Omega)\right)}^{2}\right) \\
& +\frac{\alpha}{2}\left(\left\|\nabla \hat{\mathrm{u}}^{1}\right\|_{L^{2}\left(0, T ; L^{2}(\Omega)\right)}^{2}+\left\|\nabla \hat{\mathrm{u}}^{2}\right\|_{L^{2}\left(0, T ; L^{2}(\Omega)\right)}^{2}\right) \\
& \quad \leq C\left(\epsilon, \alpha, \hat{\mathrm{v}}^{r}, \tilde{\mathrm{v}}^{r}\right) T\left(\left\|\hat{\mathrm{v}}^{1}\right\|_{L^{\infty}\left(0, T ; L^{2}(\Omega)\right)}^{2}+\left\|\hat{\mathrm{v}}^{2}\right\|_{L^{\infty}\left(0, T ; L^{2}(\Omega)\right)}^{2}\right) \\
& \quad+\epsilon\left(\left\|\nabla \hat{\mathrm{v}}^{1}\right\|_{L^{2}\left(0, T ; L^{2}(\Omega)\right)}^{2}+\left\|\nabla \hat{\mathrm{v}}^{2}\right\|_{L^{2}\left(0, T ; L^{2}(\Omega)\right)}^{2}\right),
\end{aligned}
$$

By choosing $T$ and $\epsilon$ such that $C\left(\alpha, \hat{\mathrm{v}}^{r}, \tilde{\mathrm{v}}^{r}\right) T \leq p / 4$ and $\epsilon \leq \alpha / 4$, we obtain a unique solution by Banach's fixed point theorem. The statement that $\mathrm{u}^{3} \in L^{\infty}(] 0, T\left[; H^{2}(\Omega)\right)$ is an immediate consequence of elliptic regularity theory.

\section{REFERENCES}

${ }^{1}$ A. Ajdari, "Pumping liquids using asymmetric electrode arrays,"

Physical review. E, Statistical physics, plasmas, fluids, and related interdisciplinary topics, 61, R45-8 ISSN 1063-651X.

${ }^{2}$ R. S. Eisenberg, "From structure to function in open ionic channels." The Journal of membrane biology, 171, 1-24 (1999), ISSN 0022-2631. 
New porous medium Poisson-Nernst-Planck equations

${ }^{3}$ C. L. Gardner, J. W. Jerome, and R. S. Eisenberg, "Electrodiffusion model of rectangular current pulses in ionic channels of cellular membranes," SIAM Journal on Applied, 61, 792-802 (2000).

${ }^{4}$ Z. Siwy, P. Apel, D. Dobrev, and R. Neumann, "Ion transport through asymmetric nanopores prepared by ion track etching," Science, 208, 143-148 (2003).

${ }^{5}$ L.-J. Cheng and L. J. Guo, "Ionic current rectification, breakdown, and switching in heterogeneous oxide nanofluidic devices." ACS nano, 3, 575-84 (2009), ISSN 1936-086X.

${ }^{6}$ P. Pivonka, G. A. Narsilio, R. Li, D. W. Smith, and B. Gardiner, "Electrodiffusive transport in charged porous media: From the particle-level scale to the macroscopic scale using volume averaging," Journal of Porous Media, 12, 101-118 (2009), ISSN 1091-028X.

${ }^{7}$ M. Quintard and S. Whitaker, "Transport in ordered and disordered porous media ii: Generalized volume averaging," Transport in Porous Media, 14, 179-206 (1994), ISSN 01693913, 10.1007/BF00615200.

${ }^{8}$ S. Whitaker, "Flow in porous media i: A theoretical derivation of darcy's law," Transport in Porous Media, 1, 3-25 (1986), ISSN 0169-3913, 10.1007/BF01036523.

${ }^{9}$ A. Bensoussans, J. Lions, and G. Papanicolaou, Asymptotic analysis for periodic structures (North-Holland Publishing Company, 1978) pp. 1-721.

${ }^{10}$ D. Cioranescu and P. Donato, An introduction to homogenization (Oxford University Press, 2000) ISBN 9780198565543, p. 272.

${ }^{11}$ G. A. Pavliotis and A. M. Stuart, Multiscale methods: averaging and homogenization, edited by L. Sirovich, P. Holmes, D. Barkley, M. Dellnitz, and P. Newton (2008).

${ }^{12}$ G. Allaire, A. Mikelić, and A. Piatnitski, "Homogenization of the linearized ionic transport equations in rigid periodic porous media," Journal of Mathematical Physics, 51, 123103 (2010), ISSN 00222488.

${ }^{13}$ J. R. Looker and S. L. Carnie, "Homogenization of the Ionic Transport Equations in Periodic Porous Media," Transport in Porous Media, 65, 107-131 (2006), ISSN 0169-3913.

${ }^{14}$ N. Ray, C. Eck, A. Muntean, and P. Knabner, "Variable Choices of Scaling in the Homogenization of a Nernst-Planck-Poisson Problem," preprint Nr 344 (Uni Erlangen) (2011).

${ }^{15}$ M. Schmuck, "Modeling and deriving porous media Stokes-Poisson-Nernst-Planck equations by a multiple-scale approach," Communications in Mathematical Sciences, 9, 685-710 (2011).

${ }^{16}$ E. Sanchez-Palencia, "HOMOGENIZATION IN MECHANICS: A survey of solved and 
New porous medium Poisson-Nernst-Planck equations

open problems," Rend. Sem. Mat. Univers. Politecn. Torino, 44, 1-46 (1986).

${ }^{17}$ M. Holmes, "Nonlinear Ionic Diffusion Through Charged Polymeric Gels," SIAM Journal on Applied Mathematics, 50, 839-852 (1990).

${ }^{18} \mathrm{C}$. Moyne and A. Murad, "Electro-chemo-mechanical couplings in swelling clays derived from a micro / macro-homogenization procedure," International Journal of Solids and Structures, 39, 6159-6190 (2002).

${ }^{19} \mathrm{~S}$. Torquato, Random heterogeneous materials : microstructure and macroscopic properties, Interdisciplinary applied mathematics (Springer, New York, Berlin, Heidelberg, 2002) ISBN 0-387-95167-9, numrotation dans la collection principale.

${ }^{20}$ A. Brovelli and G. Cassiani, "Effective permittivity of porous media: a critical analysis of the complex refractive index model," Geophysical Prospecting, 56, 715-727 (2008), ISSN $1365-2478$.

${ }^{21}$ K. Maex, M. R. Baklanov, D. Shamiryan, F. lacopi, S. H. Brongersma, and Z. S. Yanovitskaya, "Low dielectric constant materials for microelectronics," Journal of Applied Physics, 93, 8793-8841 (2003).

${ }^{22}$ J. L. Jackson and S. R. Coriell, "Effective Diffusion Constant in a Polyelectrolyte Solution," The Journal of Chemical Physics, 38, 959 (1963), ISSN 00219606.

${ }^{23}$ R. J. Gross and J. F. Osterle, "Membrane transport characteristics of ultrafine capillaries," The Journal of Chemical Physics, 49, 228-234 (1968).

${ }^{24}$ C. Moyne and M. A. Murad, "Macroscale modeling of cartilage: Mixture theory versus homogenization," Transport in Porous Media, 41, 215-222 (2004).

${ }^{25}$ M. Schmuck, "First error bounds for the porous media approximation of the poissonnernst-planck equations," ZAMM - Journal of Applied Mathematics and Mechanics Zeitschrift fr Angewandte Mathematik und Mechanik, 92, 304-319 (2012), ISSN 15214001.

${ }^{26}$ N. Ray, A. Muntean, and P. Knabner, "Rigorous homogenization of a stokesnernstplanckpoisson system," Journal of Mathematical Analysis and Applications, 390, 374 - 393 (2012), ISSN 0022-247X.

${ }^{27}$ L. Onsager, "Reciprocal relations in irreversible processes. i." Phys. Rev., 37, 405-26 (1931).

${ }^{28}$ L. Onsager, "Reciprocal relations in irreversible processes. ii." Phys. Rev., 38, 2265-79 (1931). 
New porous medium Poisson-Nernst-Planck equations

${ }^{29}$ M. Bazant, K. Thornton, and A. Ajdari, "Diffuse-charge dynamics in electrochemical systems," Physical Review E, 70, 1-24 (2004), ISSN 1539-3755.

${ }^{30}$ R. W. O'Brien and L. R. White, "Electrophoretic mobility of a spherical colloidal particle," Journal of the Chemical Society, Faraday Transactions 2, 74, 1607 (1978), ISSN 0300-9238.

${ }^{31}$ A. Mani and M. Z. Bazant, "Deionization shocks in microstructures." Phys Rev E, 84, 061504 (2011), ISSN 1550-2376.

${ }^{32}$ E. V. Dydek, B. Zaltzman, I. Rubinstein, D. S. Deng, A. Mani, and M. Z. Bazant, "Overlimiting current in a microchannel." Phys Rev Lett, 107, 118301 (2011), ISSN 10797114 .

${ }^{33}$ M. Schmuck and M. Z. Bazant, "Homogenization of the poisson-nernst-planck equations for ion transport in charged porous media," arXiv/1202.1916 (2012).

${ }^{34}$ A. Schmidt and K. G. Siebert, Design of Adaptive Finite Element Software The Finite Element Toolbox ALBERTA, Lecture Notes in Computational Science and Engineering, Vol. 42 (Springer, 2005).

${ }^{35}$ L. Ambrosio, N. Gigli, and G. Savaré, "Gradient flows with metric and differentiable structures, and applications to the Wasserstein space," Rend. Mat. Acc. Lincei, 15, 327$343(2004)$.

${ }^{36}$ U. Gianazza, G. Savaré, and G. Toscani, "The Wasserstein gradient flow of the Fisher information and the quantum drift-diffusion equation," Archive for rational mechanics and analysis, 194, 133-220 (2009).

${ }^{37}$ M. Herrmann and B. Niethammer, "Kramers' formula for chemical reactions in the context of Wasserstein gradient flows," Arxiv preprint arXiv:1008.3658, 1-12 (2010), arXiv:arXiv:1008.3658v2.

${ }^{38}$ R. Jordan, D. Kinderlehrer, and F. Otto, "The Variational Formulation of the FokkerPlanck Equation," SIAM Journal on Mathematical Analysis, 29, 1 (1998), ISSN 00361410.

${ }^{39}$ L. S. Bennethum, M. A. Murad, and J. H. Cushman, "Macroscale thermodynamics and the chemical potential for swelling porous media," Transport in Porous Media, 39, 187-225 (2000), ISSN 0169-3913, 10.1023/A:1006661330427.

${ }^{40}$ P. H. Nelson and S. M. Auerbach, "Self-diffusion in single-file zeolite membranes is fickian at long times," The Journal of Chemical Physics, 110, 9235-9243 (1999).

${ }^{41}$ M. Schmuck, "Aanalysis of the Navier-Stokes-Nernst-Planck-Poisson System," Mathemat- 
New porous medium Poisson-Nernst-Planck equations

ical Models and Methods in Applied Sciences, 19, 993 (2009), ISSN 0218-2025.

${ }^{42}$ E. Acerbi, G. Dal Maso, and D. Percivale, "An extension theorem from connected sets, and homogenization in general periodic domains," Nonlinear Analysis: Theory, Methods \& Applications, 18, 481-496 (1992).

${ }^{43}$ G. Allaire, A. Mikelić, and A. Piatnitski, "Homogenization approach to the dispersion theory for reactive transport through porous media," SIAM Journal of Mathematical Analysis, 42, 125-144 (2010).

${ }^{44}$ M. Castro, "Phase-field approach to heterogeneous nucleation," Physical Review B, 67. 1-8 (2003), ISSN 0163-1829.

${ }^{45}$ K. Chu and M. Bazant, "Nonlinear electrochemical relaxation around conductors," Physical Review E, 74 (2006), ISSN 1539-3755, doi:10.1103/PhysRevE.74.011501.

${ }^{46}$ Y. He, D. Gillespie, D. Boda, I. Vlassiouk, R. S. Eisenberg, and Z. S. Siwy, "Tuning transport properties of nanofluidic devices with local charge inversion." Journal of the American Chemical Society, 131, 5194-5202 (2009), ISSN 1520-5126.

${ }^{47}$ A. Roy, J. Rickman, J. Gunton, and K. Elder, "Simulation study of nucleation in a phase-field model with nonlocal interactions," Physical Review E, 57, 2610 (1998).

\section{Acknowledgement.}

Main parts of this research were conducted by the author at the Chemical Engineering Department at MIT and supported by the Swiss National Science Foundation (SNSF) under the prospective researcher Grant PBSKP2-12459/1. 